DOI: $10.1002 /(($ please add manuscript number $))$

Article type: Communication

\title{
Metal-organic framework-derived hybrid carbon nanocages as bifunctional electrocatalyst for oxygen reduction and evolution
}

Shaohong Liu, ${ }^{a}{ }^{\dagger}$ Zhiyu Wang, ${ }^{a}{ }^{\dagger}$ Si Zhou, ${ }^{b, \dagger}$ Fengjiao Yu, ${ }^{c}$ Mengzhou Yu, ${ }^{a}$ Chang-Yang Chiang, ${ }^{c}$ Wuzong Zhou, ${ }^{c}$ Jijun Zhao ${ }^{b, *}$ and Jieshan Qiu ${ }^{a, *}$

Dr. S. H. Liu, Prof. Z. Y. Wang, Dr. S. Zhou, F. J. Yu, M. Z. Yu, C. Chiang, Prof. W. Z. Zhou, Prof. J. J. Zhao, Prof. J. S. Qiu

${ }^{a}$ State Key Lab of Fine Chemicals, Carbon Research Laboratory, Liaoning Key Lab for Energy Materials and Chemical Engineering, School of Chemical Engineering, Dalian University of Technology, Dalian 116024, China.

${ }^{\mathrm{b}}$ Education Ministry Key Lab of Materials Modification by Laser, Ion and Electron Beams, Dalian University of Technology, Dalian 116024, China

${ }^{c}$ School of Chemistry, University of St Andrews, St Andrews, KY16 9ST, United Kingdom

${ }^{\dagger}$ These authors contribute equally to this work.

E-mail: jqiu@dlut.edu.cn, zhaojj@dlut.edu.cn

Keywords: oxygen electrocatalysis, bifunctional electrocatalyst, metal-organic frameworks, hollow nanostructure, first-principles calculation

Abstract Oxygen reduction reaction (ORR) and oxygen evolution reaction (OER) is cornerstone reactions of many renewable energy technologies. Developing cheap yet durable substitutes of precious metal catalysts, especially the bifunctional electrocatalysts with high activity for both reactions and their streamlined coupling process, are highly desirable to reduce the processing cost and complexity of renewable energy systems. Herein we report a facile strategy for synthesizing double-shelled hybrid nanocages with outer shells of Co-N-doped graphitic carbon (Co-NGC) and inner shells of $\mathrm{N}$-doped microporous carbon (NC) by templating against core-shelled metal-organic frameworks. The double-shelled NC@Co-NGC nanocages well integrate the high activity of Co-NGC shells into robust NC hollow framework with enhanced diffusion kinetics, exhibiting superior electrocatalytic properties to $\mathrm{Pt}$ and $\mathrm{RuO}_{2}$ as bifunctional electrocatalyst for ORR and OER and holding the promise as efficient air electrode catalysts in $\mathrm{Zn}$-air batteries. First-principles calculations revealed that high catalytic activities of Co-NGC shells are due to the synergistic electron transfer and redistribution 
between Co nanoparticles, the graphitic carbon and the doped N species. Strong yet favorable adsorption of $\mathrm{OOH}^{*}$ intermediate on high density of uncoordinated hollow-site $\mathrm{C}$ atoms with respect to Co lattice in Co-NGC structure is a vital rate-determining step to achieve excellent bifunctional electrocatalytic activity.

Oxygen reduction reaction (ORR) and oxygen evolution reaction (OER) are the cornerstone of many renewable energy storage and conversion technologies such as metal-air batteries, fuel cells and water electrolysis. ${ }^{[1-3]}$ Both reactions, however, need highly active catalysts to achieve high efficiency since the oxygen electrode is a strongly irreversible system associated with high activation overpotential and sluggish kinetics. Noble metals (e.g., Pt) and their oxides (e.g., $\mathrm{RuO}_{2}, \mathrm{IrO}_{2}$ ) have been found to be the most active catalysts for electrocatalytic reduction and evolution of molecular oxygen. However, their large-scale application is greatly prohibited by high cost, supply scarcity and inferior durability. ${ }^{[4,5]}$ On the other hand, as the universal choice of ORR catalyst, the OER activity of $\mathrm{Pt}$ is limited by the in situ formation of insulating platinum oxides in the process. $\mathrm{IrO}_{2}$ and $\mathrm{RuO}_{2}$ are unstable at high potentials due to the in situ transformation to higher-valent oxides, in spite of the highest activity towards OER. ${ }^{[6]}$ To fulfill the demands in practical use, the development of low-cost yet durable bifunctional electrocatalysts with high activity towards both ORR and OER process is highly desired to reduce the cost and complexity of the renewable energy storage and conversion systems.

Recent studies highlighted that transitional metal-N-doped carbon nanohybrids (M-N-C, M $=\mathrm{Fe}, \mathrm{Co}, \mathrm{Ni}$, etc.) hold promise as substitutes of noble metal electrocatalysts in both acidic and alkaline medium. ${ }^{[7-9]}$ In such catalysts, the presence of transitional metals helps to greatly improve the crystallinity and electrical conductivity of carbon matrix by catalytic graphitization upon preparation at high temperature, which in turn function to protect the metals from corrosion and aggregation during the electrochemical reactions. ${ }^{[10,11]}$ More importantly, the interaction and synergy of metal species, the doped $\mathrm{N}$ species and carbon lattice creates 
sufficient localized reactive sites by modifying the charge distribution on carbon surface via the promoted electron transfer effect, which changes the local work function for $\mathrm{O}_{2}$ adsorption and consequently facilitate the ORR or OER. ${ }^{[12,13]}$ Very recently, the synergistic effect of metal@C nanoparticles and neighboring metal- $\mathrm{N}_{x}$ coordination sites has been demonstrated to promote the $\mathrm{O}_{2}$ adsorption by overlapping the highest occupied molecular orbital (HOMO) and lowest unoccupied molecular orbital (LUMO) of triplet $\mathrm{O}_{2}$ molecule during ORR. ${ }^{[14]}$ Because of these combined effects, some exciting performance that is comparable or even superior to noble metal catalysts, has been achieved due to the enhanced reaction kinetics and structural stability in MN-C electrocatalyst. Among available strategies, direct pyrolysis of metal-organic frameworks (MOFs) composed of atomically dispersed metal-containing clusters between organic linkers, represents one of the most efficient approaches for producing nanostructured $\mathrm{M}-\mathrm{N}-\mathrm{C}$ catalysts with uniformly distributed active sites, highly controlled dimensions and porosity. ${ }^{[15-19]}$ However, only a handful of them show the capability of catalyzing more than one electrochemical reactions under appropriate conditions, especially for the reverse ORR/OER pair reactions. ${ }^{[20-24]}$ To tackle these issues, one need to know how to design and fabricate highperformance ORR/OER electrocatalysts with multifunctional electrocatalytic activities, meanwhile, to understand the origin of their intrinsic activity and the catalytic reaction pathways involved in the process.

Since the electrocatalytic reduction and evolution of oxygen occurs at the electrodeelectrolyte- $\mathrm{O}_{2}$ triple-phase interface, engineering the nanoarchitecture of the $\mathrm{M}-\mathrm{N}-\mathrm{C}$ electrocatalysts is critical to generate highly exposed active surface for fast exchange and reaction of the reactants (e.g., $\mathrm{O}_{2}, \mathrm{H}^{+}, \mathrm{OH}^{-}$or $\mathrm{H}_{2} \mathrm{O}$ ). Hollow nanostructures with low density, very thin shells with a thickness below tens of nanometers and high permeability provide an ideal framework for better controlling the local chemical micro-environment of the electrocatalysts upon reaction. The formation of hollow cavity inside catalyst nanoparticle not only provides extra triple-phase interface to accelerate oxygen reduction and evolution, but also 
ensures fast diffusion of the reactant across the catalyst even if the aggregation occurs. ${ }^{[25,26]}$ Macroscopically, it manifests as effective utilization of active mass due to high accessibility of the inner pores to surrounding reactants. In the present work, we report the fabrication of carbon-based hybrid double-shelled nanocages (DSNCs) derived from core-shelled zeolite imidazole frameworks (ZIFs), a well-known subfamily of MOFs, as an active and stable bifunctional electrocatalyst for both ORR and OER. ${ }^{[27]}$ The hybrid nanocages feature with closely packed double shells: a ZIF-8 derived inner shell of N-doped microporous carbon (NC) and a ZIF-67 derived outer shell of Co-decorated N-doped mesoporous graphitic carbon (CoNGC), denoted as NC@Co-NGC DSNCs. In this kind of novel structure, the ZIF-67 derived Co-NGC shells with stable structure and good conductivity is highly active to catalyze the ORR/OER while the ZIF-8 derived NC act as the nanostructured hollow framework to facilitate the diffusion kinetics. ${ }^{[28,29]}$ Their heterogeneous hybridization renders superior bifunctional catalytic properties and durability to noble metal catalysts (e.g., Pt and $\mathrm{RuO}_{2}$ ) towards ORR/OER and their coupled process, which are hardly attainable by the catalysts from single MOF precursor. First-principles calculations have revealed that the Co-NGC shells owe their highly catalytic activity to the charge transfer and redistribution between Co nanoparticles, the graphitic carbon and the $\mathrm{N}$ dopants. This facilitates the favorable chemisorption of $\mathrm{OOH}^{*}$, the rate-determining intermediate for ORR and OER, on uncoordinated $\mathrm{C}$ atoms on Co-NGC surface, enabling the bifunctional electrocatalytic activity of NC@Co-NGC DSNC catalyst. The potential and superior performance of NC@Co-NGC DSNC electrocatalyst are also demonstrated in primary and rechargeable Zn-air batteries operated in air.

The synthetic strategy of NC@Co-NGC DSNCs is schematically illustrated in Figure 1. Uniform polyhedral nanocrystals of ZIF-8 were first fabricated by the coordination of $\mathrm{Zn}^{2+}$ and 2-MeIm in methanol. ${ }^{[30]}$ They function as the crystal seeds for subsequent epitaxial growth of ZIF-67 with similar isoreticular structure $\left(\left[\mathrm{M}(\mathrm{MeIm})_{2}\right](\mathrm{M}=\mathrm{Zn}\right.$ for ZIF-8 or Co for ZIF-67)) and unit cell parameters $(a$ ZIF-8 $=16.9910 \AA, a$ ZIF-67 $=16.9589 \AA)$ in the presence of $\mathrm{Co}^{2+}$ and 
2-MeIm in methanol at room temperature. XRD analysis shows that the resultant ZIF-8@ZIF67 sample reasonably share similar feature with ZIF-8 and ZIF-67 crystals in peak intensity and position (Figure S1a). The successful growth of ZIF-67 on ZIF-8 crystals can be visualized by distinct color change from white to violet in the suspension after the seed-mediated growth (see inset of Figure S1a). A panoramic view by filed-emission scanning electron microscopy (FESEM) reveals that the sample consists of entirely uniform rhombic dodecahedrons with a smooth surface and an average edge length of ca. $550 \mathrm{~nm}$. The precisely controlled epitaxial growth enables the ZIF-8@ZIF-67 crystals to inherit the polyhedral shape of ZIF-8 seeds that are enclosed by twelve $<110>$ facets (Figure S1b and Figure S2a). No aggregates or free ZIF67 particles are observed in the samples due to efficient deposition of ZIF-67 on ZIF-8 crystals. Transmission electron microscopy (TEM) characterization further confirms the formation of polyhedral particles with high uniformity (Figure S1c). It should be noted that it is hard to distinguish the interface between ZIF-67 shell and ZIF-8 core, as well as the thickness of ZIF67 shell due to their similar lattice structure. However, the formation of ZIF-8@ZIF-67 coreshelled structure can be clearly visualized by elemental mapping, where the region of $\mathrm{Zn}$ element is closely surrounded by that of Co element (Figure S1d). The presence of C and N element is also detected in ZIF-8@ZIF-67 nanocrystals. The molar ratio of Zn to Co is 2.63 and the $\mathrm{N}$ content can be as high as 29 at.\%, confirmed by energy-dispersive X-ray (EDX) analysis (Figure S3).

The core-shelled ZIF-8@ZIF-67 particles are then subjected to thermal annealing in flowing $\mathrm{N}_{2}$ at $800{ }^{\circ} \mathrm{C}$ at a ramp rate of $2{ }^{\circ} \mathrm{C} \min ^{-1}$, followed by etching away the unstable Co nanoparticles with $\mathrm{H}_{2} \mathrm{SO}_{4}$. Surprisingly, well-defined hollow nanocages instead of solid nanoparticles commonly reported in literature up to now are created by the decomposition and carbonization of ZIF-8@ZIF-67 crystals. ${ }^{[31]}$ The FESEM analysis shows that the as-synthesized samples have inherited the polyhedral shape of the parent ZIF-8@ZIF-67 crystals without collapse (Figure 2a and $\mathbf{b}$ ). The average edge length of the particles is slightly reduced to $c a$. 
500-520 nm due to the shrinkage to denser carbon-based composites. The particles have a rough surface anchored with numerous short carbon nanotubes (CNTs), as shown in Figure 2c. The formation of CNTs is the result of the catalytic effect of Co nanoparticles on particle surface. ${ }^{[1]}$ FESEM image of a broken particle clearly indicates the presence of inner cavities underneath the carbon shells (Figure 2c). The structure of closely packed double shells with uniform thickness is visualized by TEM characterization (Figure $2 \mathbf{d}$ and e). The ZIF-8 derived inner shells are composed of dense amorphous carbon with an average thickness of $c a .60 \mathrm{~nm}$, which are formed by the decomposition of $\mathrm{ZIF}-8$ to $\mathrm{ZnO} /$ carbon composite and subsequent carbothermal reduction to $\mathrm{Zn}$ /carbon composite, followed by the vaporization of $\mathrm{Zn}$ with the temperature rise. ${ }^{[31]}$ The ZIF-67-derived outer shells consist of relatively loose carbon with an average thickness of $c a .40 \mathrm{~nm}$, from which many short carbon nanotubes with a length of tens of nanometers are extruded (Figure 2e and Figure S4). HRTEM analysis reveals the formation of graphitic carbon structures decorated with Co nanoparticles enclosed in several graphitic carbon layers, as a result of the catalytic graphitization of amorphous carbon by Co nanoparticles of several nanometers at high temperature (Figure 2f). ${ }^{[16,28,32]}$ The Co nanoparticles in graphitic carbon sheath are very stable against acidic etching, which is desired to enhance the electrocatalytic stability. By varying the size of ZIF-8 seeds employed, both the size and interior volume of the nanocages could be rationally tailored. This is evidenced by the fact that uniform NC@Co-NGC DSNCs with various edge lengths of $c a .150,300$ to $750 \mathrm{~nm}$ can be fabricated by a similar approach except using ZIF-8 nanocrystals with different sizes as the seeds ( Figure 2g-i).

The structural characteristics of NC@Co-NGC DSNCs are identified by XRD analysis (Figure 3a). The relatively sharp peak at $26^{\circ}$ corresponds to (002) plane of graphitic carbon while the others at around $44^{\circ}, 51^{\circ}$ and $76^{\circ}$ is attributed to the diffraction from (111), (200) and (220) planes of metallic Co $(a=3.545 \AA$, JCPDS No. 15-0806). Figure S5 shows the Raman spectra of NC@Co-NGC DSNCs, ZIF-67 derived Co-NGC and ZIF-8 derived NC. For 
NC@Co-NGC and Co-NGC, the G bands at around $1589 \mathrm{~cm}^{-1}$ corresponds to the in-plane vibration of $\mathrm{sp}^{2}$ carbon while the $\mathrm{D}$ band at around $1338 \mathrm{~cm}^{-1}$ mainly reflects the structural defects in graphitic structure. The intensity ratio between them $\left(\mathrm{I}_{\mathrm{D}} / \mathrm{I}_{\mathrm{G}}\right)$ is around 0.96 , revealing the domination of short-range order graphitic structure. Compared to $\mathrm{NC}$, the $\mathrm{G}$ band of NC@Co-NGC and Co-NGC is upshifted by $12 \mathrm{~cm}^{-1}$ in wavelength, suggesting strong electronic interaction in them, possibly between carbon and metal atoms. Thermogravimetric analysis (TGA) reveals that the content of metallic Co in NC@Co-NGC DSNCs is 16.8 wt.\% (Figure S6). The chemical composition and the effect of $\mathrm{N}$ doping are investigated by $\mathrm{X}$-ray photoelectron spectroscopy (XPS), revealing the presence of $\mathrm{C}, \mathrm{N}, \mathrm{O}$ and Co elements in NC@Co-NGC DSNCs, where the N content is calculated to be 5.28 at.\% (Figure 3b). The high-resolution $\mathrm{N}$ 1s spectrum reveals the presence of two types of $\mathrm{N}$ species, namely, the pyridinic N (ca. $398.4 \mathrm{eV}$ ) and graphitic N (ca. $400.9 \mathrm{eV})$ in the nanocages (Figure 3c). The Co $2 p$ XPS spectrum is resolved into two pairs of $2 \mathrm{p}_{3 / 2} / 2 \mathrm{p}_{1 / 2}$ doublets for metallic Co $(778.0 / 792.6 \mathrm{eV})$ and $\mathrm{N}$-coordinated $\mathrm{Co}^{2+}\left(\mathrm{Co}^{-} \mathrm{N}_{x}\right)(780.7 / 795.3 \mathrm{eV})$ with an energy separation of $14.6 \mathrm{eV}$ (Figure 3d). ${ }^{[33,34]}$ The C1s XPS spectrum can be deconvoluted into the peaks for $s p^{2}$-hydridized carbon $(284.6 \mathrm{eV}), \mathrm{C}-\mathrm{O}$ bonds $(286.5 \mathrm{eV})$ and $\mathrm{O}-\mathrm{C}=\mathrm{O}$ group $(288.8 \mathrm{eV})$, as shown in Figure S7a. ${ }^{[35]}$ The identification of C-N bonds at $285.1 \mathrm{eV}$ confirms the $\mathrm{N}$ doping in NC@Co-NGC DSNCs. ${ }^{[35]}$ The presence of O signal can be attributed to residual oxygen in ZIFderived carbon and the adsorbed oxygen from environmental atmosphere (Figure S7b). The $\mathrm{N}_{2}$ adsorption/desorption isotherms of NC@Co-NGC DSNCs are type-I isotherms (Figure 3e), in which the isotherms with sharp increase in volume at low relative pressure indicate the presence of micropores, mainly from ZIF-8 derived NC shells. The hysteresis loop at relative pressure between 0.5-0.9 suggests the co-existence of mesopores from Co-decorated graphitic carbon shell derived from ZIF-67. The pore size distribution indicates that the micropores are smaller than $1 \mathrm{~nm}$ in size, while the mesopores are below $70 \mathrm{~nm}$ (Figure 3f). The as-synthesized 
NC@Co-NGC DSNCs with unique hollow structure and hierarchical porosity has a BrunauerEmmett-Teller (BET) surface area of $c a .476 \mathrm{~m}^{2} \mathrm{~g}^{-1}$ and total pore volume of $0.303 \mathrm{~m}^{3} \mathrm{~g}^{-1}$.

The formation of double-shelled NC@Co-NGC nanocages is believed to be governed by a surface-stabilized contraction process originated from the thermal stability difference of ZIF67 and ZIF-8. TGA analysis reveals that ZIF-67 is less stable than ZIF-8 (Figure S8), and would decompose first to form rigid shells of $\mathrm{CoO} /$ carbon composite around $\mathrm{ZIF}-8$ cores at above 500 ${ }^{\circ} \mathrm{C}$ (Figure S9a, e and Figure S10). Then, the rigid $\mathrm{CoO} /$ carbon shells could generate an outward adhesive force at the interface between them and ZIF-8 cores, which suppress the inward contraction of ZIF-8 due to the thermal-induced polycondensation. As a result, hollow cavities are formed inside ZIF-8 cores as a balance of the mass loss (mainly the small molecules such as $\mathrm{NH}_{3}$ and $(\mathrm{CN})_{2}$ ) from ZIF-8 decomposition at evaluated temperature (e.g., $550{ }^{\circ} \mathrm{C}$ ) (Figure S9b and f). ${ }^{[36]}$ At higher temperature (e.g., 600-700 ${ }^{\circ} \mathrm{C}$ ), the $\mathrm{CoO}$ in outer shells is reduced to metallic Co by surrounding carbonaceous species formed in situ to catalyze the formation of graphitic carbon (Figure S9c, g and Figure S10). Eventually, double-shelled NC@Co-NGC nanocages are obtained after the evaporation of nanosized $\mathrm{Zn}$ at $700-800{ }^{\circ} \mathrm{C}$ (Figure 3a, Figure S9d and h, Figure S10). Without stabilization by ZIF-67 shells, only solid carbon particles with shrunken volume would be formed due to the inward contraction of ZIF8 under identical conditions. In this case, the vacancies generated by mass loss are largely squeezed out of the particles by contraction-induced stress (Figure S11). To confirm the surface-stabilized contraction mechanism proposed, core-shelled ZIF-67@ZIF-8 polyhedrons are fabricated and annealed at $800{ }^{\circ} \mathrm{C}$. As expected, only solid Co-NGC@NC nanoparticles instead of hollow structure are yielded in this case because of the lower stability of ZIF-67 cores than that of ZIF-8 shells (Figure S12). It should be noted that the reported non-equilibrium heterogeneous contraction is not applied for the formation of NC@Co-NGC nanocages because a slow ramp rate of $2{ }^{\circ} \mathrm{C} \mathrm{min}^{-1}$ is unlikely to reach a sharp temperature gradient within the particles in this process. ${ }^{[37]}$ 
The electrocatalytic activity of NC@Co-NGC DSNCs towards ORR was first identified by cyclic voltammograms $(\mathrm{CV})$ tests conducted in $\mathrm{Ar}$ or $\mathrm{O}_{2}$ - saturated $0.1 \mathrm{M} \mathrm{KOH}$ electrolyte at a scan rate of $10 \mathrm{mV} \mathrm{s}^{-1}$ (Figure 4a). A substantial reduction process is found to occur at ca. 0.81 $\mathrm{V}$ versus reversible hydrogen electrode (RHE) in $\mathrm{O}_{2}$-saturated $0.1 \mathrm{M} \mathrm{KOH}$ solution, whereas featureless quasi-rectangular voltammogram is observed within the same voltage window in Ar-saturated electrolyte. To gain deeper insight into electrocatalytic activity and kinetics of NC@Co-NGC DSNCs towards ORR, linear sweep voltammograms (LSVs) are recorded on a rotating disk electrode $(\mathrm{RDE})$ a rotation rate of $1600 \mathrm{rpm}$ in $\mathrm{O}_{2}$-saturated $0.1 \mathrm{M} \mathrm{KOH}$ electrolyte at a scan rate of $10 \mathrm{mV} \mathrm{s}^{-1}$ (Figure 4b). The curves of NC@Co-NGC DSNC electrodes show obvious plateau with an onset potential of $c a .0 .92 \mathrm{~V}$ and a half-wave potential $\left(E_{1 / 2}\right)$ of $0.82 \mathrm{~V}$, which is comparable to those of $\mathrm{Pt} / \mathrm{C}\left(0.96 \mathrm{~V}, E_{1 / 2}=0.82 \mathrm{~V}\right)$, and outperform most previously reported carbon-based ORR catalysts (Table S1). Moreover, the unique and wide current plateau from 0.7 to $0 \mathrm{~V}$ observed in the case of the NC@Co-NGC DSNC electrode represents a diffusion-controlled process corresponding to the efficient four-electron-dominated ORR pathway. ${ }^{[38]}$ To demonstrate the advantage of NC@Co-NGC DSNCs in oxygen electrocatalysis, the ORR performance of solid NC, Co-NGC and Co-NGC@NC particles is also investigated under identical conditions (Figure S13 and Figure S14a). In comparison with NC@Co-NGC DSNCs, they exhibit higher onset overpotential ( $0.33 \mathrm{~V}$ for Co-NGC, $0.36 \mathrm{~V}$ for $\mathrm{NC}, 0.34 \mathrm{~V}$ for Co-NGC@NC) and half-wave potential (0.8 V for Co-NGC, $0.73 \mathrm{~V}$ for NC, $0.79 \mathrm{~V}$ for CoNGC@NC), as can be seen in Figure 4b and Figure S14a. The superior catalytic activity of NC@Co-NGC DSNCs towards ORR is further verified by the Tafel plots obtained from the polarization curves. The NC@Co-NGC DSNC has a Tafel slope of $51 \mathrm{mV} \mathrm{dec}{ }^{-1}$, much lower

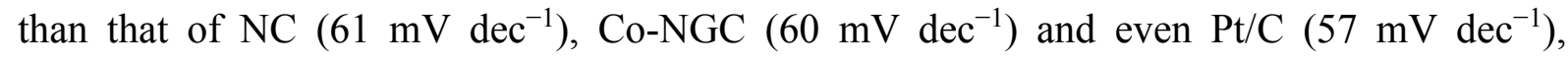
suggesting the favourable ORR kinetics in the NC@Co-NGC DSNCs electrocatalyst with a unique hollow nanostructure (Figure 4c). The apparent electrochemical surface area (ECSA) of NC@Co-NGC, Co-NGC and NC is compared by measuring their double-layer capacitance 
$\left(C_{\mathrm{dl}}\right)$, which is typically used to represent the ECSA (Figure S15). ${ }^{[39-41]}$ The NC@Co-NGC possesses over $60 \%$ higher $C_{\mathrm{dl}}$ than that of Co-NGC, revealing the positive effect of hollow nanostructure on electrocatalytic activity.

The electron transfer number per oxygen molecule (n) and kinetic limiting current density $\left(J_{\mathrm{k}}\right)$ for ORR is determined from the LSV curves (Figure S16) according to the Koutechy-Levich (K-L) equation. The K-L plots of NC@Co-NGC DSNCs manifest good parallel linearity over the potential ranging from 0.71 to $0.46 \mathrm{~V}$, suggesting first-order reaction kinetics for ORR (Figure 4d). ${ }^{[42]}$ It is noted that the NC@Co-NGC DSNCs exhibit a similar slope with that of $\mathrm{Pt} / \mathrm{C}$ electrodes. The $\mathrm{n}$ is determined to be $\sim 4.0$, indicating that the ORR proceeds via a fourelectron-transfer scheme involving the in situ $\mathrm{OH}^{-}$formation in alkaline medium, which are further confirmed by the rotating ring-disk electrode (RRDE) measurements (Figure S16). ${ }^{[43-}$ 45] The NC@Co-NGC DSNC catalyst still shows an equal $J_{\mathrm{k}}$ of $12.8 \mathrm{~mA} \mathrm{~cm}{ }^{-2}$ to that of Pt/C, which is much higher than that of $\mathrm{NC}\left(3.2 \mathrm{~mA} \mathrm{~cm}^{-2}\right)$ and Co-NGC $\left(7.9 \mathrm{~mA} \mathrm{~cm}^{-2}\right)$ catalyst. (Figure S17). In comparison with Pt/C catalyst, the NC@Co-NGC DSNC catalyst exhibits superior durability and higher resistance to the methanol cross-over effect in oxygen-saturated 0.1 M KOH (Figure S18). Furthermore, the NC@Co-NGC DSNC catalyst also shows excellent ORR activity in acidic medium. They exhibit much lower onset overpotential $(0.5 \mathrm{~V})$ than that of Co-NGC $(0.57 \mathrm{~V})$ and $\mathrm{NC}(0.63 \mathrm{~V})$ electrodes, as well as superior stability to commercial $\mathrm{Pt} / \mathrm{C}$ catalysts (Figure $\mathbf{S 1 9}$ ).

The oxygen evolution activities of NC@Co-NGC DSNC catalysts are also evaluated in $\mathrm{O}_{2}$ saturated 0.1 M KOH solution with $i R$-correction. The NC@Co-NGC DSNC catalyst reaches a current density of $10 \mathrm{~mA} \mathrm{~cm}^{-2}$ (a value expected for a $10 \%$ efficient solar water-splitting device) at a potential $\left(E_{j=10}\right)$ of $1.64 \mathrm{~V}$, which is compares favorably to that of $\mathrm{RuO}_{2}(1.60 \mathrm{~V})$, but much lower than those of Co-NGC (1.70 V), Co-NGC@NC (1.68 V), Pt/C (1.82 V) and other reported catalysts (Figure 5a, Figure S14b and Table S1). Also, NC@Co-NGC DSNC catalysts exhibit a much lower Tafel slope $\left(91 \mathrm{mV} \mathrm{dec}^{-1}\right)$ with respect to $\mathrm{NC}\left(115 \mathrm{mV} \mathrm{dec}^{-1}\right)$, 
Co-NGC (100 mV dec $\left.{ }^{-1}\right), \mathrm{RuO}_{2}\left(110 \mathrm{mV} \mathrm{dec}{ }^{-1}\right)$ and $\mathrm{Pt} / \mathrm{C}\left(171 \mathrm{mV} \mathrm{dec}^{-1}\right)$ (Figure 5b), suggesting the favorable OER kinetics in hollow nanostructured electrocatalyst. Much enhanced electrocatalytic activities are also observed for NC@Co-NGC DSNC electrodes in 1 $\mathrm{M} \mathrm{KOH}$ electrolyte, where the onset potential is close to that of $\mathrm{RuO}_{2}$ catalyst (Figure $\mathbf{S 2 0 a}$ ). The stability test results demonstrate that the NC@Co-NGC DSNC catalyst is very stable for the OER, whereas $\mathrm{RuO}_{2}$ exhibits a rapid voltage increase at the constant current of $10 \mathrm{~mA} \mathrm{~cm}^{-}$ 2 in short time in $0.1 \mathrm{M} \mathrm{KOH}$ (Figure S20b).

The pyrolysis temperature is a critical factor in terms of tuning the activity of NC@Co-NGC DSNC electrocatalysts due to its significant effect on the chemical composition and structure of the ZIF-derived products. As discussed above, the NC@Co-NGC DSNC made at $800{ }^{\circ} \mathrm{C}$ due to the formation of Co-NGC shells exhibits the highest current density and the lowest overpotential for ORR and OER (Figure S21). Lower annealing temperature (e.g., 600-700 ${ }^{\circ} \mathrm{C}$ ), however, yields the poor conductive composites made of carbon and Co nanoparticles of several nanometers as a result of inadequate graphitization process and carbonthermal reduction of metal oxides, accompanied with lower specific surface area $\left(359 \mathrm{~m}^{2} \mathrm{~g}^{-1}\right)$ (Figure S8, Figure S22a-c and Figure S23). While annealing at higher temperature (e.g., $900{ }^{\circ} \mathrm{C}$ ) is not favorable for retaining high $\mathrm{N}$-doping level in whole architecture and high specific surface area as a result of the destruction of micropores (Figure S22d, e and Figure S23). In this case, the Co particles with much larger size of tens to a hundred nanometer are yielded due to strong mobility and severe fusion of metal particles, which are less stable and can be easily removed by acidic etching (Figure S22f). Meanwhile, the $\mathrm{N}$ content is also reduced to 4 at.\% by increasing the annealing temperature from 800 to $900{ }^{\circ} \mathrm{C}$, and the atomic ratio of pyridinic $\mathrm{N}$ in total $\mathrm{N}$ is decreased from 70 to $45 \%$ (Figure S24d). The loss of $\mathrm{N}$ and stable Co in the hybrids significantly reduces the charge transfer and delocalization on carbon lattice and more importantly, weakens the interaction and electron transfer between carbon and metal atoms (to be further discussed below in detail), thereby being unfavorable for the chemisorption of 
reactive intermediates on large ECSA that help to accelerate the ORR and OER. ${ }^{[12,45]}$. In the sample annealed at $800{ }^{\circ} \mathrm{C}$, the ratio of Co-NGC in NC@Co-NGC DSNCs also affects their electrocatalytic performance. The NC@Co-NGC DSNCs with 16.8 wt.\% Co shows the lowest overpotential to the benchmark nanocage catalysts with lower (e.g., 9.6 wt.\%) or higher (e.g., 28.2 wt.\%) Co content towards ORR/OER because of more electrochemical active phase than the former but higher specific surface area than that of the latter (Figure S25-27).

The application potential of the NC@Co-NGC DSNC materials as bifunctional oxygen electrocatalysts has been explored, and the overall oxygen electrode activity is evaluated by the difference of OER and ORR metrics $\left(\Delta E=E_{j=10}-E_{1 / 2}\right)$. Remarkably, the NC@Co-NGC DSNCs exhibit a $\Delta E$ of $0.82 \mathrm{~V}$, much lower than that of $\mathrm{Pt} / \mathrm{C}(0.94 \mathrm{~V}), \mathrm{RuO}_{2}(1.0 \mathrm{~V})$ and CoNGC $(0.95 \mathrm{~V})$, as well as most of the bifunctional catalysts reported to date such as metal-free carbon hybrids (e.g., N, S-carbon nanosheet, $\Delta E=0.88 \mathrm{~V}$ ) and transition metal oxides (e.g., $\left.\mathrm{MnCo}_{2} \mathrm{O}_{4} / \mathrm{N}-\mathrm{CNT}, \Delta E=1.02 \mathrm{~V}\right)\left(\right.$ Figure 5c and Table S1). ${ }^{[38],[46]}$ Motivated by the superior catalytic activity of the NC@Co-NGC DSNCs for both ORR and OER, the proof-to-concept tests were conducted to demonstrate the feasibility of NC@Co-NGC DSNCs as the air cathode catalyst in primary Zn-air batteries (Figure S28a). The open-circuit voltage of the Zn-air battery with NC@Co-NGC DSNC electrode is $1.45 \mathrm{~V}$, which is comparable to the batteries with Pt/C electrode (1.46 V) but higher than that of $\mathrm{RuO}_{2}$ electrode (1.31 V) (Figure S28b). Three cointype Zn-air batteries with NC@Co-NGC DSNC catalysts can be connected in series to light a light-emitting diode (LED) array panel $(11 \times 44,3.7 \mathrm{~V})$ (Figure S28c). The batteries with NC@Co-NGC DSNCs as cathode catalyst exhibit high capacities of ca. 617, 602, 565, and 537 $\mathrm{mA} \mathrm{h} \mathrm{gZn}^{-1}$ at the current densities of 2, 5, 10 and $20 \mathrm{~mA} \mathrm{~cm}^{-2}$, respectively (Figure S28d). These values are higher than that of the primary batteries with $\mathrm{Pt} / \mathrm{C}$ catalyst, which deliver lower capacities of ca. 580, 535, 510 and $495 \mathrm{~mA} \mathrm{~h} \mathrm{gz}^{-1}$ at identical current densities (Figure S28e). The peak power density of the Zn-air battery with NC@Co-NGC DSNC electrode is $109 \mathrm{~mW}$ $\mathrm{cm}^{-2}$ (Figure S29). The potential of NC@Co-NGC DSNC catalyst in rechargeable Zn-air 
batteries is evaluated using identical cell configuration except add $\mathrm{Zn}(\mathrm{Ac})_{2}(0.2 \mathrm{M})$ into $6 \mathrm{M}$ $\mathrm{KOH}$ electrolyte (Figure S30a). The charging and discharging polarization curves of rechargeable Zn-air batteries with NC@Co-NGC DSNC, Pt/C or $\mathrm{RuO}_{2}$ as cathode catalysts are shown in Figure S30b. The NC@Co-NGC DSNC catalyst exhibits comparable discharging performance to $\mathrm{Pt} / \mathrm{C}$ catalyst at both low (e.g., $20 \mathrm{~mA} \mathrm{~cm}^{-2}$ ) and high current densities (e.g., 80 $\mathrm{mA} \mathrm{cm}{ }^{-2}$ ), but significantly outperforms $\mathrm{RuO}_{2}$ catalysts in rechargeable $\mathrm{Zn}$-air batteries. Upon charging, the NC@Co-NGC DSNC catalyst shows substantially lower overpotential for driving OER than that of $\mathrm{Pt} / \mathrm{C}$ and $\mathrm{RuO}_{2}$ catalysts at the current densities of up to $40 \mathrm{~mA} \mathrm{~cm}^{-2}$, showing reliable bifunctionally electrocatalytic properties. The rechargeable $\mathrm{Zn}$-air batteries with NC@Co-NGC DSNC catalyst exhibit superior lifetime (56 h) and discharging voltage retention (77.5\%) to that with $\mathrm{Pt} / \mathrm{C}(14 \mathrm{~h}, 50 \%)$ or $\mathrm{RuO}_{2}(33 \mathrm{~h}, 59 \%)$ catalyst upon discharging-charging at a current density of $10 \mathrm{~mA} \mathrm{~cm}^{-2}$, showing good reversibility and durability (Figure S30c-e). This performance may be further enhanced by further optimizing the cell configuration, e.g., the three-electrode cells that OER and ORR electrocatalysts are loaded onto two separate electrodes for charge and discharge, ${ }^{[45,47]}$ which can prevent the bifunctional catalyst from coming into contact with the oxidative (or reductive) potential during ORR (or OER).

Apparently, the Co-NGC shells in NC@Co-NGC DSNCs contribute largely to the catalytic activities for both ORR and OER while the hollow nanostructure accelerates the reaction kinetics. To gain atomistic insight into the origin of bifunctional electrochemical activity, firstprinciples calculation in terms of density function theory (DFT) methods is performed to determine the active sites and catalytic reactions over Co-NGC shells. As the radius of realistic Co-embedded N-doped graphitic carbon structures in Co-NGC shells attains several nanometers, a slab model instead of cluster model is used to represent the Co-NGC surface where ORR and OER are predominant. It consists of $\mathrm{N}$-doped graphene monolayer adsorbed on (111) surface of the face-centered cubic $(f c c)$ Co crystal (Figure 6a). The (111) plane of $f c c$ Co is adopted because of its best compatibility for coupling with graphene layer. Their in-plane 
cell parameters are very close with lattice mismatch below $1 \%$. Consequently, Co-NGC comprises of two types of $\mathrm{C}$ sites: on right top or hollow sites with respect to the Co lattice (denoted as the top-site $\mathrm{C}$ and hollow-site $\mathrm{C}$, respectively), and the former is coordinated with underlying Co atoms. According to the experimental results, pyridinic and graphitic-type $\mathrm{N}$ atoms are uniformly substituted into graphene at a doping level of $c a .6$ at.\%. Two models of Co-NGC structure are thus considered in terms of the location of $\mathrm{N}$ dopants: on the right top or hollow sites with respect to the Co lattice (denoted as model A and B, respectively). Mulliken charge analysis indicates little difference between the two models, which both present electron transfer of $0.26 e$ from each Co atom to the graphitic layer, giving 0.15 and $0.1 e$ to the top-site $\mathrm{C}$ and hollow-site $\mathrm{C}$ atoms, respectively. The $\mathrm{N}$ dopants in the graphitic sheets further induce charge redistribution to the $\mathrm{C}$ atoms nearby, which directly affect their electrocatalytic performance to be discussed below.

The key to high activity of a bifunctional ORR/OER electrocatalyst is the favorable strength of the interaction with the reaction intermediates involved in both reactions. The weak adsorption of the intermediates on active sites results in low efficiency of the reaction, while too strong binding between them starves one of or even all the reactions by blocking the catalytic surface. To work out the ORR and OER activities of the Co-NGC catalysts, the adsorption free energy of the oxygenated intermediate species, including the $\mathrm{OOH}^{*}, \mathrm{OH}^{*}$ and $\mathrm{O}^{*}$, involved in ORR and OER at different $\mathrm{C}$ sites in two Co-NGC models is calculated. The overpotentials of ORR $\left(\eta^{\mathrm{ORR}}\right)$ and OER $\left(\eta^{\mathrm{OER}}\right)$ are then determined by the standard hydrogen electrode (SHE) method. ${ }^{[48]}$ For comparison, the model of an isolated N-doped graphitic carbon sheet (NGC) with the same scale is also constructed to calculate the ORR/OER overpotentials (Figure 6a). In alkaline solution, ORR and OER occur via the four electron reaction paths, listed as equations (S5) to (S12) in Supporting Information. The $\eta^{\mathrm{ORR}}$ versus the adsorption free energy of $\mathrm{OOH}^{*}\left(\Delta G_{\mathrm{OOH}^{*}}\right)$ for various reactive sites on Co-NGC surface of both models presents a volcano-type plot (Figure 6b). Benefited from the electron transfer effect, the 
adsorption of $\mathrm{OOH}^{*}$ on carbon surface of Co-NGC structure is greatly enhanced, as indicated by the much lower adsorption free energy $\left(\Delta G_{\mathrm{OOH}^{*}}\right)$ at different $\mathrm{C}$ sites $(3.9-4.9 \mathrm{eV})$ than that of the NGC without Co involved (4.9 - $5.8 \mathrm{eV})$. Specifically, the hollow-site C atoms with strong bonding with $\mathrm{OOH}^{*}$ exhibit much lower $\eta^{\mathrm{ORR}}$ of $0.477-0.68 \mathrm{~V}$ than that of the top-site $\mathrm{C}$ atoms $\left(\eta^{\mathrm{ORR}}=0.87-1.23 \mathrm{~V}\right)$ coordinated to underlying Co. For the $\mathrm{C}$ sites in close proximity to doped $\mathrm{N}$ atoms, the bonding with oxygenated species is too strong (Mulliken charge of +0.06 e) to suppress the further reaction, thus leading to high $\eta^{\mathrm{ORR}}$ of $0.81-0.98 \mathrm{~V}$. Among the hollowsite $\mathrm{C}$ atoms, those para to graphitic $\mathrm{N}$ in model $\mathrm{A}$ (Mulliken charge of $-0 .-09 e$ ) exhibit the lowest $\eta^{\text {ORR }}$ of $0.477 \mathrm{~V}$, approaching the value of Pt identified theoretically $(0.45 \mathrm{~V}) .{ }^{[49]} \mathrm{In}$ contrast, the NGC model shows very high $\eta^{\mathrm{ORR}}$ of above $1.24 \mathrm{~V}$ due to the weak bonding of $\mathrm{OOH}^{*}$ species over the carbon surface. For the OER process, the hollow-site $\mathrm{C}$ atoms exhibit the strongest adsorption of $\mathrm{OOH}^{*}$ relative to $\mathrm{O}^{*}$ species and high catalytic activity with $\eta^{\mathrm{OER}}$ of $0.388-0.74 \mathrm{~V}$, which is superior to that of top-site $\mathrm{C}$ atoms $\left(\eta^{\mathrm{OER}}=0.82-1.48 \mathrm{~V}\right)$ and the $\mathrm{C}$ atoms in close proximity to doped $\mathrm{N}$ atoms $\left(\eta^{\mathrm{OER}}=1.56-1.65 \mathrm{~V}\right)$ on Co-NGC surface (Figure 6c). A lowest $\eta^{\text {OER }}$ of $0.388 \mathrm{~V}$ can be achieved at the hollow-site $\mathrm{C}$ atoms para to pyridinic $\mathrm{N}$ in model A (Mulliken charge of $-0.1 e$ ), which is even lower than the theoretical value $\mathrm{RuO}_{2}(0.42$ $\mathrm{V})^{[50]}$. In the case of $\mathrm{NGC}$, the $\mathrm{C}$ atoms in close proximity to doped $\mathrm{N}$ atoms have the lowest $\eta^{\mathrm{OER}}$ of $0.5 \mathrm{~V}$ and $\eta^{\mathrm{ORR}}$ of $1.2 \mathrm{~V}$, and the rest shows high overpotentials of over $1.1-1.67 \mathrm{~V}$ for both reactions. ${ }^{[51,52]}$ The highly active hollow-site $\mathrm{C}$ atoms towards ORR/OER $\left(\eta^{\text {ORR/OER }}<0.65\right.$ V) account for $47 \%$ of the total $\mathrm{C}$ atoms in the Co-NGC structure, which endows the catalyst with high efficiency towards oxygen electrocatalysis.

The elementary reactions of ORR and OER over the identified active sites of Co-NGC structure are further investigated at various electrode potential $(U)$. At $U=0$, the overall reaction for the four electron transfer steps is exothermic by $1.844 \mathrm{eV}$ in alkaline media with $\mathrm{pH}=13$ for ORR (Figure 6d). The equilibrium potential is $1.844 / 4=0.461 \mathrm{~V}$, at which the overall reaction has free energy change equal to zero. At $U=0.461 \mathrm{~V}$, an energy barrier of $0.478 \mathrm{eV}$ 
has to be overcome for the formation of $\mathrm{OOH}^{*}$. By applying a negative voltage $U=-0.017 \mathrm{~V}$ to the electrodes, all the reaction steps become downhill and can occur spontaneously, which corresponds to a ORR overpotential of $0.478 \mathrm{~V}$. For the OER, the opposite process of ORR applies. At equilibrium potential $U=0.461 \mathrm{~V}$, the formation of $\mathrm{OOH}^{*}$ from $\mathrm{O}^{*}$ and $\mathrm{OH}^{-}$species are associated with the highest energy barrier of $0.387 \mathrm{eV}$ (Figure 6e). This energy barrier disappears at $U=0.848 \mathrm{~V}$, determining an OER overpotential of $0.387 \mathrm{~V}$. Apparently, this leads one to conclude that the formation of $\mathrm{OOH}^{*}$ could be identified as the rate-limiting step for ORR and OER over Co-NGC structure in alkaline media. The strong but favorable adsorption of the $\mathrm{OOH}^{*}$ intermediates on uncoordinated hollow-site $\mathrm{C}$ atoms in Co-NGC structure is an essential rate-determining step to achieve high bifunctional electrocatalytic activity for both the ORR and OER reactions.

In summary, we have developed a facile approach to prepare hybrid carbon nanocages with $\mathrm{N}$-doped carbon (NC) as inner shells and Co-N-doped graphitic carbon (Co-NGC) as outer shells by the controlled pyrolysis of core-shelled ZIF-8@ZIF-67 crystals. The unusual formation of hollow nanostructures is attributed to surface-stabilized contraction of coreshelled ZIF crystals at high temperature. The integration of Co-NGC shells into robust hollow NC structure renders the double-shelled NC@Co-NGC nanocages with high activity and enhanced diffusion kinetics for electrochemical oxygen reduction and evolution. As the electrocatalyst, NC@Co-NGC nanocages exhibit superior electrocatalytic activity and durability in comparison to $\mathrm{Pt}$ and $\mathrm{RuO}_{2}$ catalysts towards ORR and OER, respectively, and hold potential as the bifunctional air electrode catalysts in rechargeable Zn-air batteries with a high peak power density of $109 \mathrm{~mW} \mathrm{~cm}^{-2}$, high capacities at the current density of 2-20 mA $\mathrm{cm}^{-2}$ and long lifetime. The first-principles calculation reveals that the strong yet favorable adsorption of the rate-determining intermediate $\left(\mathrm{OOH}^{*}\right)$ on high density of uncoordinated hollow-site $\mathrm{C}$ atoms with respect to Co lattice may account for the bifunctional electrocatalytic activity of the NC@Co-NGC nanocage catalysts towards ORR and OER. In particular, the 
active $\mathrm{C}$ sites at the para-position of doped graphitic-type and pyridinic-type $\mathrm{N}$ atoms exhibit the highest activity towards ORR and OER, respectively. The present work may shed a new insight into the design and fabrication of highly active M-N-C electrocatalysts with tuned chemical nature, morphology and electrocatalytic bifunctions for both ORR and OER.

\section{Supporting Information}

Supporting Information is available from the Wiley Online Library or from the author.

\section{Acknowledgements}

This study is partly supported by the National Natural Science Foundation of China (Nos. 51522203, 21336001 and 21361162004). ZW acknowledges the support from the Recruitment Program of Global Youth Experts, Fok Ying Tung Education Foundation (No. 151047) and Xinghai Scholarship of Dalian University of Technology. WZ thanks EPSRC for financial support to the electron microscopy facility (EP/F019580/1).

Received: ((will be filled in by the editorial staff))

Revised: ((will be filled in by the editorial staff)) Published online: ((will be filled in by the editorial staff))

[1] P. G. Bruce, S. A. Freunberger, L. J. Hardwick, J.-M. Tarascon, Nat. Mater. 2012, 11, 19.

[2] M. K. Debe, Nature 2012, 486, 43.

[3] J. Suntivich, K. J. May, H. A. Gasteiger, J. B. Goodenough, Y. Shao-Horn, Science 2011, 334, 1383.

[4] G. Wu, P. Zelenay, Acc. Chem. Res. 2013, 46, 1878.

[5] J.-I. Jung, H. Y. Jeong, J.-S. Lee, M. G. Kim, J. Cho, Angew. Chem. Int. Ed. 2014, 53, 4582.

[6] T. Maiyalagan, K. A. Jarvis, S. Therese, P. J. Ferreira, A. Manthiram, Nat. Commun. 2014, $5,3949$.

[7] J. Masa, W. Xia, M. Muhler, W. Schuhmann, Angew. Chem. Int. Ed. 2015, 54, 10102.

[8] M. Zeng, Y. Liu, F. Zhao, K. Nie, N. Han, X. Wang, W. Huang, X. Song, J. Zhong, Y. Li, Adv. Funct. Mater. 2016, 26, 4397.

[9] G. Wu, K. L. More, C. M. Johnston, P. Zelenay, Science 2011, 332, 443.

[10] N. L. Torad, R. R. Salunkhe, Y. Li, H. Hamoudi, M. Imura, Y. Sakka, C.-C. Hu, Y. Yamauchi, Chem. Eur. J. 2014, 20, 7895.

[11] Y. Liu, H. Jiang, Y. Zhu, X. Yang, C. Li, J. Mater. Chem. A 2016, 4, 1694.

[12] D. Guo, R. Shibuya, C. Akiba, S. Saji, T. Kondo, J. Nakamura, Science 2016, 351, 361.

[13] D. Deng, L. Yu, X. Chen, G. Wang, L. Jin, X. Pan, J. Deng, G. Sun, X. Bao, Angew. Chem. Int. Ed. 2013, 52, 371.

[14] W.-J. Jiang, L. Gu, L. Li, Y. Zhang, X. Zhang, L.-J. Zhang, J.-Q. Wang, J.-S. Hu, Z. Wei, L.-J. Wan, J. Am. Chem. Soc. 2016, 138, 3570.

[15] K. S. Park, Z. Ni, A. P. Cote, J. Y. Choi, R. Huang, F. J. Uribe-Romo, H. K. Chae, M. O'Keeffe, O. M. Yaghi, Proc. Natl. Acad. Sci. U. S. A. 2006, 103, 10186.

[16] B. Y. Xia, Y. Yan, N. Li, H. B. Wu, X. W. Lou, X. Wang, Nat. Energy 2016, 1, 15006.

[17] W. Xia, A. Mahmood, R. Zou, Q. Xu, Energy Environ. Sci. 2015, 8, 1837.

[18] X. Li, Q. Sun, J. Liu, B. Xiao, R. Li, X. Sun, J. Power Sources 2016, 302, 174. 
[19] Y. Zhao, Z. Song, X. Li, Q. Sun, N. Cheng, S. Lawes, X. Sun, Energy Storage Mater. 2016, 2,35 .

[20] R. Wu, X. Qian, X. Rui, H. Liu, B. Yadian, K. Zhou, J. Wei, Q. Yan, X.-Q. Feng, Y. Long, L. Wang, Y. Huang, Small 2014, 10, 1932.

[21] L. Hou, L. Lian, L. Zhang, G. Pang, C. Yuan, X. Zhang, Adv. Funct. Mater. 2015, 25, 238.

[22] F. Wu, J. T. Lee, F. Fan, N. Nitta, H. Kim, T. Zhu, G. Yushin, Adv. Mater. 2015, 27, 5579.

[23] X.-Y. Yu, L. Yu, H. B. Wu, X. W. Lou, Angew. Chem. Int. Ed. 2015, 54, 5331.

[24] Z. Song, N. Cheng, A. Lushington, X. Sun, Catalysts 2016, 6, 116.

[25] J. Liu, S. Z. Qiao, J. S. Chen, X. W. Lou, X. R. Xing, G. Q. Lu, Chem. Commun. 2011, 47, 12578.

[26] P. M. Arnal, M. Comotti, F. Schuth, Angew. Chem. Int. Ed. 2006, 45, 8224.

[27] J. S. Qiu, Z. Y. Wang, S. H. Liu, Chinese Patent, State Intellectual Property Office of the P.R. China 28.07.2015, application No. 201510448674.2.

[28] J. Tang, R. R. Salunkhe, J. Liu, N. L. Torad, M. Imura, S. Furukawa, Y. Yamauchi, J. Am. Chem. Soc. 2015, 137, 1572.

[29] Y.-Z. Chen, C. Wang, Z.-Y. Wu, Y. Xiong, Q. Xu, S.-H. Yu, H.-L. Jiang, Adv. Mater. 2015, 27, 5010.

[30] R. Wu, X. Qian, K. Zhou, J. Wei, J. Lou, P. M. Ajayan, ACS Nano 2014, 22, 6297.

[31] H. B. Wu, S. Wei, L. Zhang, R. Xu, H. H. Hng, X. W. Lou, Chem. Eur. J. 2013, 19, 10804.

[32] R. Wu, D. P. Wang, X. Rui, B. Liu, K. Zhou, A. W. K. Law, Q. Yan, J. Wei, Z. Chen, Adv. Mater. 2015, 27, 3038.

[33] W.-B. Luo, X.-W. Gao, S.-L. Chou, J.-Z. Wang, H.-K. Liu, Adv. Mater. 2015, 27, 6862.

[34] Q. Lin, X. Bu, A. Kong, C. Mao, F. Bu, P. Feng, Adv. Mater. 2015, 27, 3431.

[35] Z. Wang, Y. Dong, H. Li, Z. Zhao, H. Bin Wu, C. Hao, S. Liu, J. Qiu, X. W. Lou, Nat. Commun. 2014, 5, 5002.

[36] F. Zheng, Y. Yang, Q. Chen, Nat. Commun. 2014, 5, 5261.

[37] J. G. Guan, F. Z. Mou, Z. G. Sun, W. D. Shi, Chem. Commun. 2010, 46, 6605.

[38] K. Qu, Y. Zheng, S. Dai, S. Z. Qiao, Nano Energy 2016, 19, 373.

[39] C. C. L. McCrory, S. Jung, J. C. Peters, T. F. Jaramillo, J. Am. Chem. Soc. 2013, 135, 16977.

[40] Z.-Q. Liu, H. Cheng, N. Li, T. Y. Ma, Y.-Z. Su, Adv. Mater. 2016, 28, 3777.

[41] H. B. Wu, B. Y. Xia, L. Yu, X.-Y. Yu, X. W. Lou, Nat. Commun. 2015, 6, 6512.

[42] Y. Hou, T. Huang, Z. Wen, S. Mao, S. Cui, J. Chen, Adv. Energy Mater. 2014, 4, 1400337.

[43] S. Guo, S. Zhang, L. Wu, S. Sun, Angew. Chem. Int. Ed. 2012, 51, 11770.

[44] Y. Liang, Y. Li, H. Wang, J. Zhou, J. Wang, T. Regier, H. Dai, Nat. Mater. 2011, 10, 780.

[45] J. Zhang, Z. Zhao, Z. Xia, L. Dai, Nat. Nanotechnol. 2015, 10, 444.

[46] A. Zhao, J. Masa, W. Xia, A. Maljusch, M.-G. Willinger, G. Clavel, K. Xie, R. Schlögl, W. Schuhmann, M. Muhler, J. Am. Chem. Soc. 2014, 136, 7551.

[47] Y. Li, M. Gong, Y. Liang, J. Feng, J. E. Kim, H. Wang, G. Hong, B. Zhang, H. Dai, Nat. Commun. 2013, 4, 1805.

[48] J. Rossmeisl, Z. W. Qu, H. Zhu, G. J. Kroes, J. K. Nørskov, J. Electroanal. Chem. 2007, $607,83$.

[49] J. K. Nørskov, J. Rossmeisl, A. Logadottir, L. Lindqvist, J. R. Kitchin, T. Bligaard, H. Jónsson, J. Phys. Chem. B 2004, 108, 17886.

[50] I. C. Man, H.-Y. Su, F. Calle-Vallejo, H. A. Hansen, J. I. Martínez, N. G. Inoglu, J. Kitchin, T. F. Jaramillo, J. K. Nørskov, J. Rossmeisl, ChemCatChem 2011, 3, 1159.

[51] H. B. Yang, J. Miao, S. F. Hung, J. Chen, H. B. Tao, X. Wang, L. Zhang, R. Chen, J. Gao, H. M. Chen, L. Dai, B. Liu, Sci. Adv. 2016, 2, e1501122.

[52] C. Tang, H.-F. Wang, X. Chen, B.-Q. Li, T.-Z. Hou, B. Zhang, Q. Zhang, M.-M. Titirici, F. Wei, Adv. Mater. 2016, 28, 6845. 


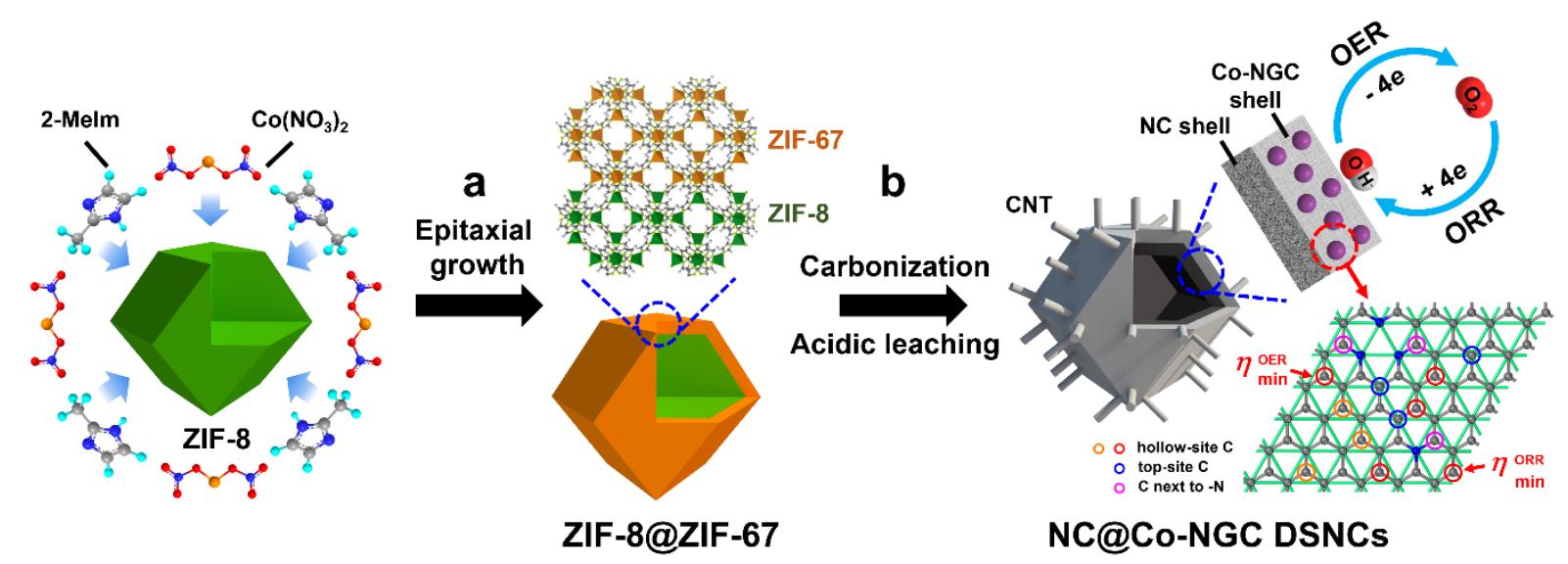

Figure 1. Schematic illustration of the synthesis of NC@Co-NGC DSNCs. (a) The growth of core-shell structured ZIF-8@ZIF-67 crystals by epitaxial growth of ZIF-67 on pre-formed ZIF8 polyhedral nanoparticles. (b) The formation of NC@Co-NGC DSNCs by annealing the coreshelled ZIF-8@ZIF-67 crystals in flowing $\mathrm{N}_{2}$ at $800{ }^{\circ} \mathrm{C}$, followed by acid washing. 


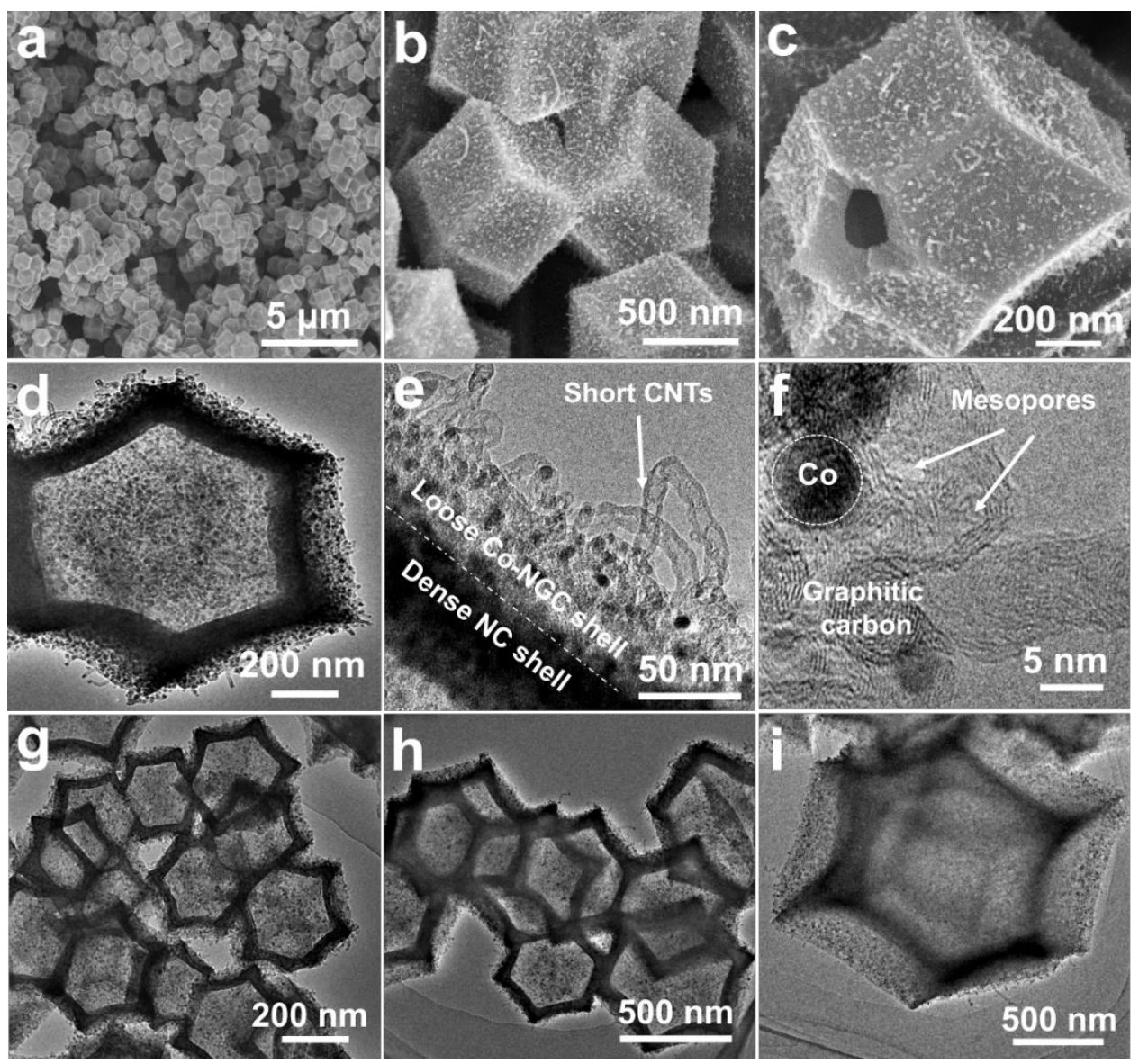

Figure 2. $(a, b)$ FESEM images of NC@Co-NGC DSNCs. (c) FESEM image of a cracked nanocage with exposed interior. (d) TEM image of a NC@Co-NGC nanocage. (e) TEM image showing the presence of closely packed NC inner shell and Co-NGC outer shell anchored with short CNTs in the nanocages. (f) HRTEM image showing the formation of Co nanoparticles encapsulated in graphitic carbon shells. (g-i) TEM images of NC@Co-NGC DSNCs with various edge length of ca. 150, 300 to $750 \mathrm{~nm}$, respectively. 

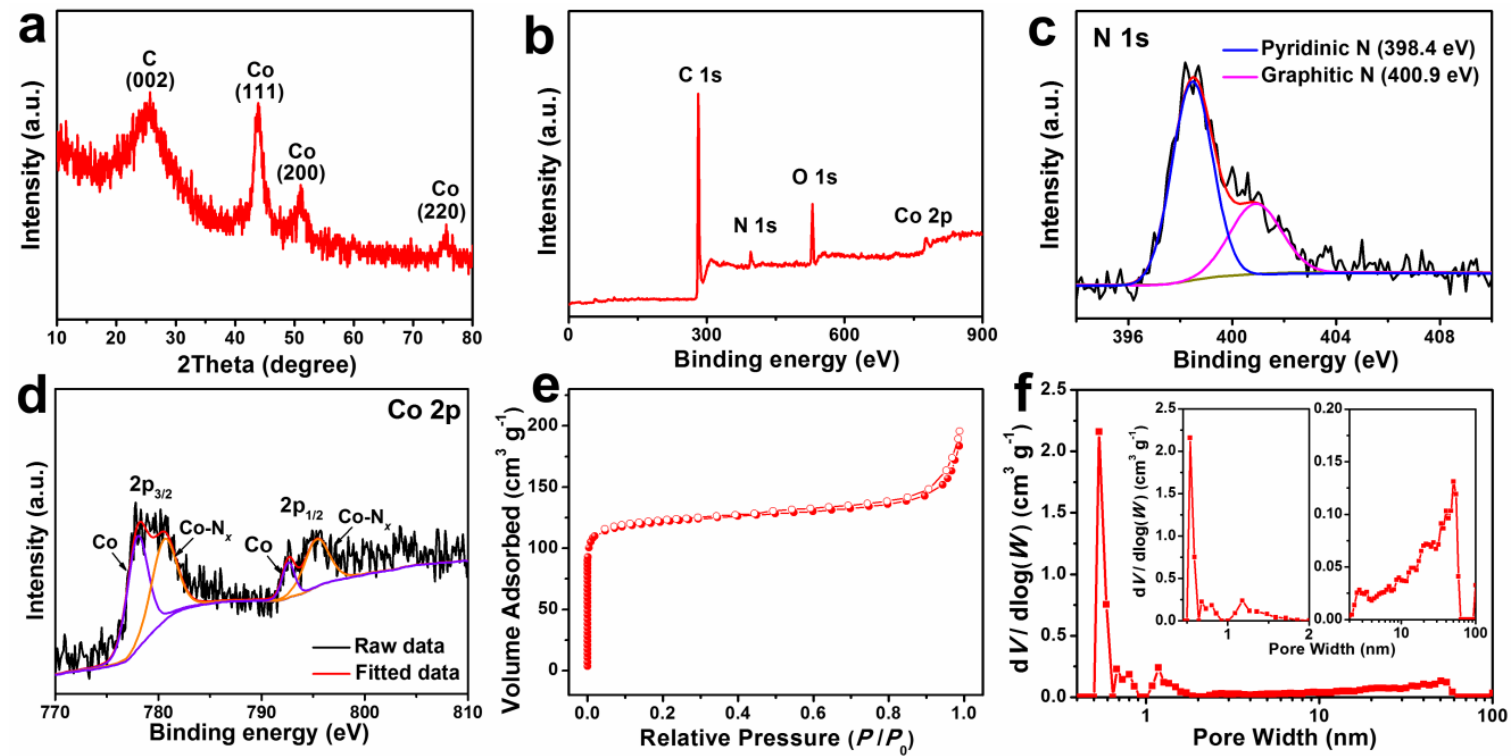

Figure 3. (a) XRD pattern, (b) XPS full scan, (c) N 1s XPS spectrum, (d) Co 2p XPS spectrum, and (e) $\mathrm{N}_{2}$ adsorption-desorption isotherm of NC@Co-NGC DSNCs. (f) Pore size distribution, showing the co-existence of micropores and mesopores in NC@Co-NGC nanocages. 

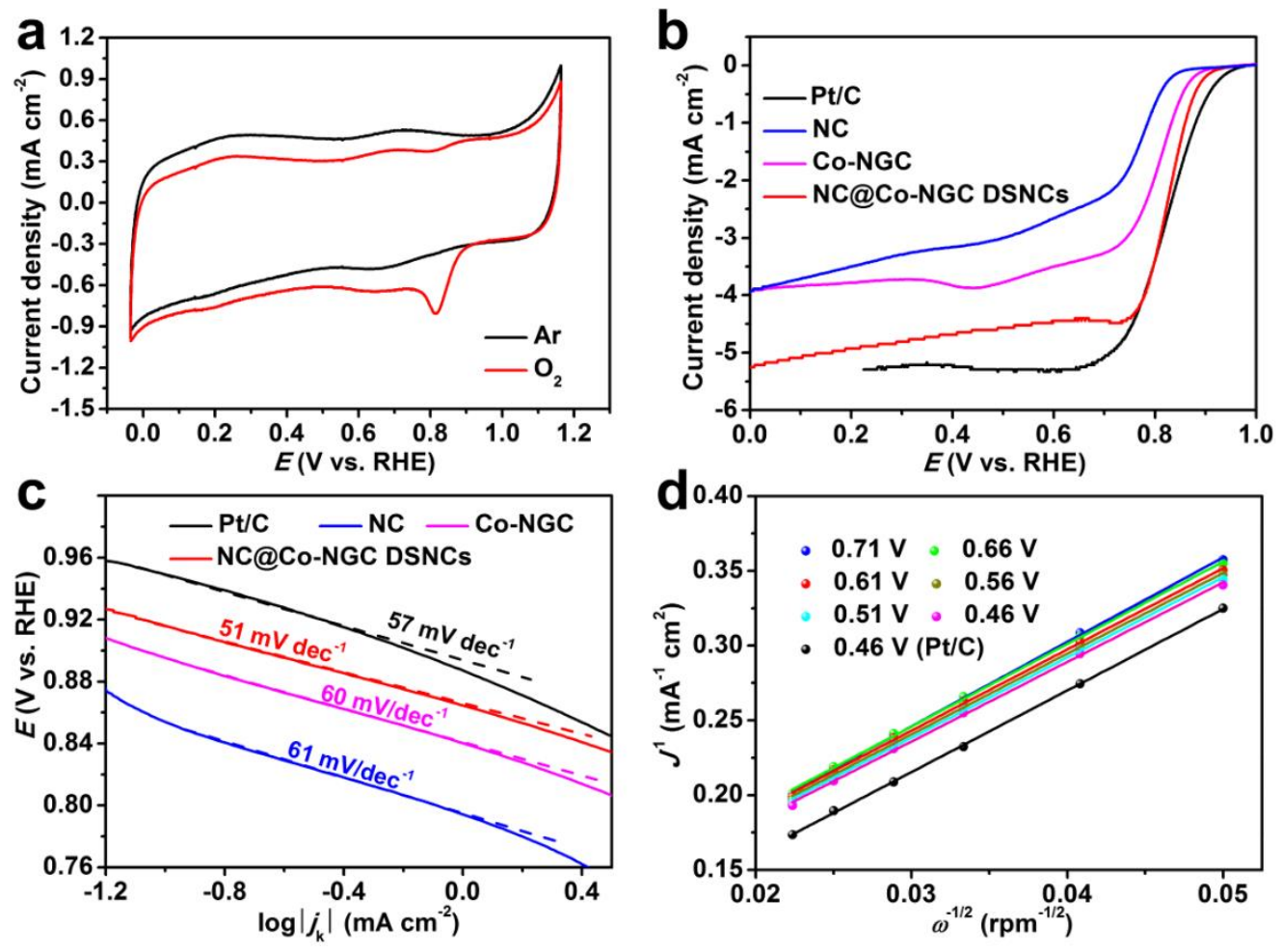

Figure 4. (a) CVs of NC@Co-NGC DSNC catalysts in $0.1 \mathrm{M} \mathrm{KOH}$ solution saturated by Ar or $\mathrm{O}_{2}$ at a scan rate of $10 \mathrm{mV} \mathrm{s}^{-1}$. (b) ORR polarization curves of NC, Co-NGC, NC@Co-NGC DSNC, and $\mathrm{Pt} / \mathrm{C}$ catalysts at a rotation rate of $1600 \mathrm{rpm}$ in $\mathrm{O}_{2}$-saturated $0.1 \mathrm{M} \mathrm{KOH}$ solution at a scan rate of $10 \mathrm{mV} \mathrm{s}^{-1}$. (c) Tafel plots of NC, Co-NGC, NC@Co-NGC DSNC, and Pt/C catalysts. (d) Koutecky-Levich plots of NC@Co-NGC DSNC and Pt/C catalysts at various potentials. 

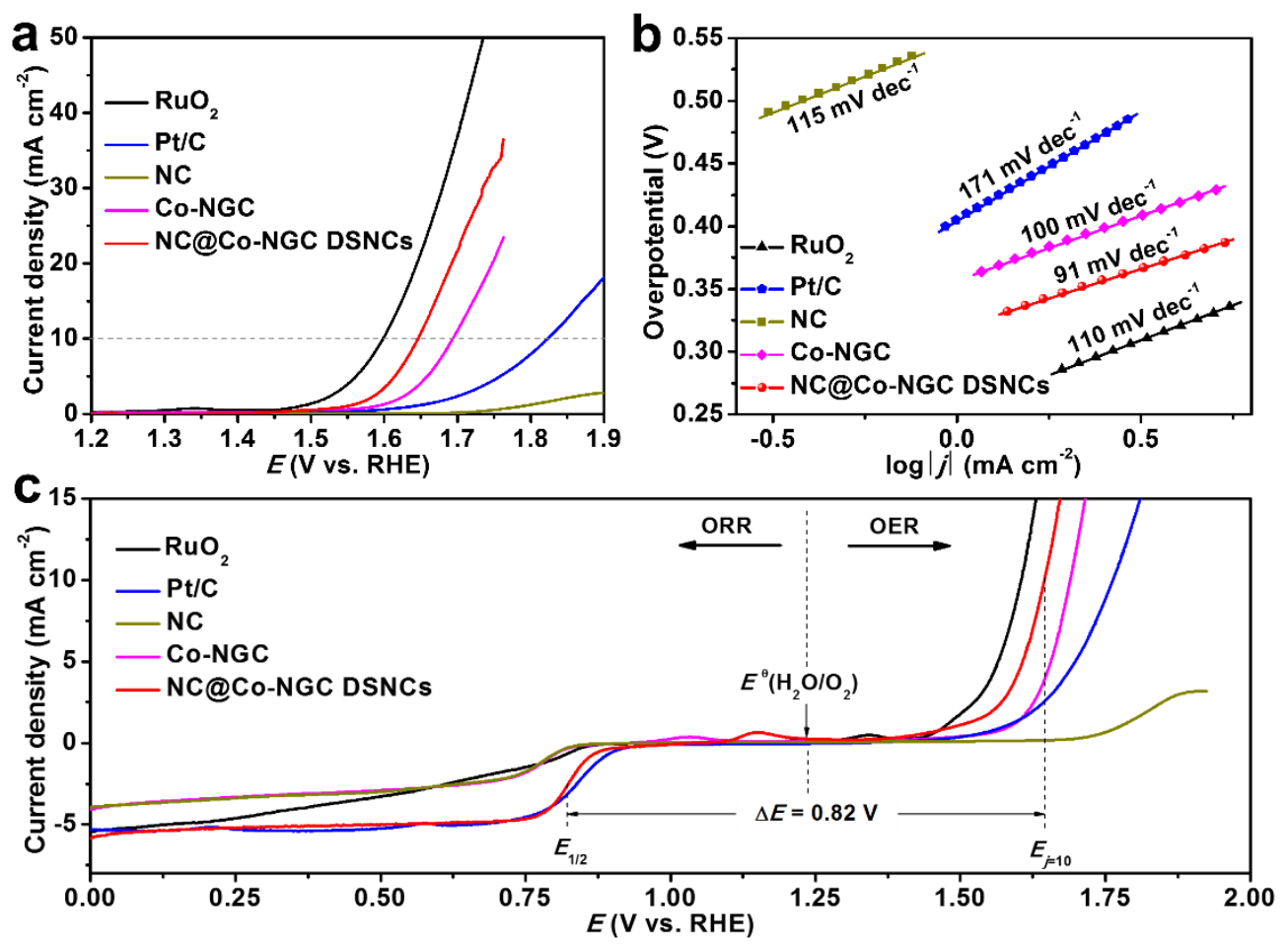

Figure 5. (a) $i R$-corrected OER polarization curves of NC, Co-NGC, NC@Co-NGC DSNC, $\mathrm{Pt} / \mathrm{C}$, and $\mathrm{RuO}_{2}$ catalysts in $\mathrm{O}_{2}$-saturated $0.1 \mathrm{M} \mathrm{KOH}$ solution at a scan rate of $10 \mathrm{mV} \mathrm{s}^{-1}$ at 1600 rpm. (b) Tafel plots of NC, Co-NGC, NC@Co-NGC DSNC, Pt/C, and RuO 2 catalysts derived from OER polarization curves. (c) IR-corrected polarization curves of NC, Co-NGC, NC@Co-NGC DSNC, Pt/C, and $\mathrm{RuO}_{2}$ catalysts, which are tested by using the three-electrode system in the full OER/ORR region in $\mathrm{O}_{2}$-saturated $0.1 \mathrm{M} \mathrm{KOH}$ solution. 


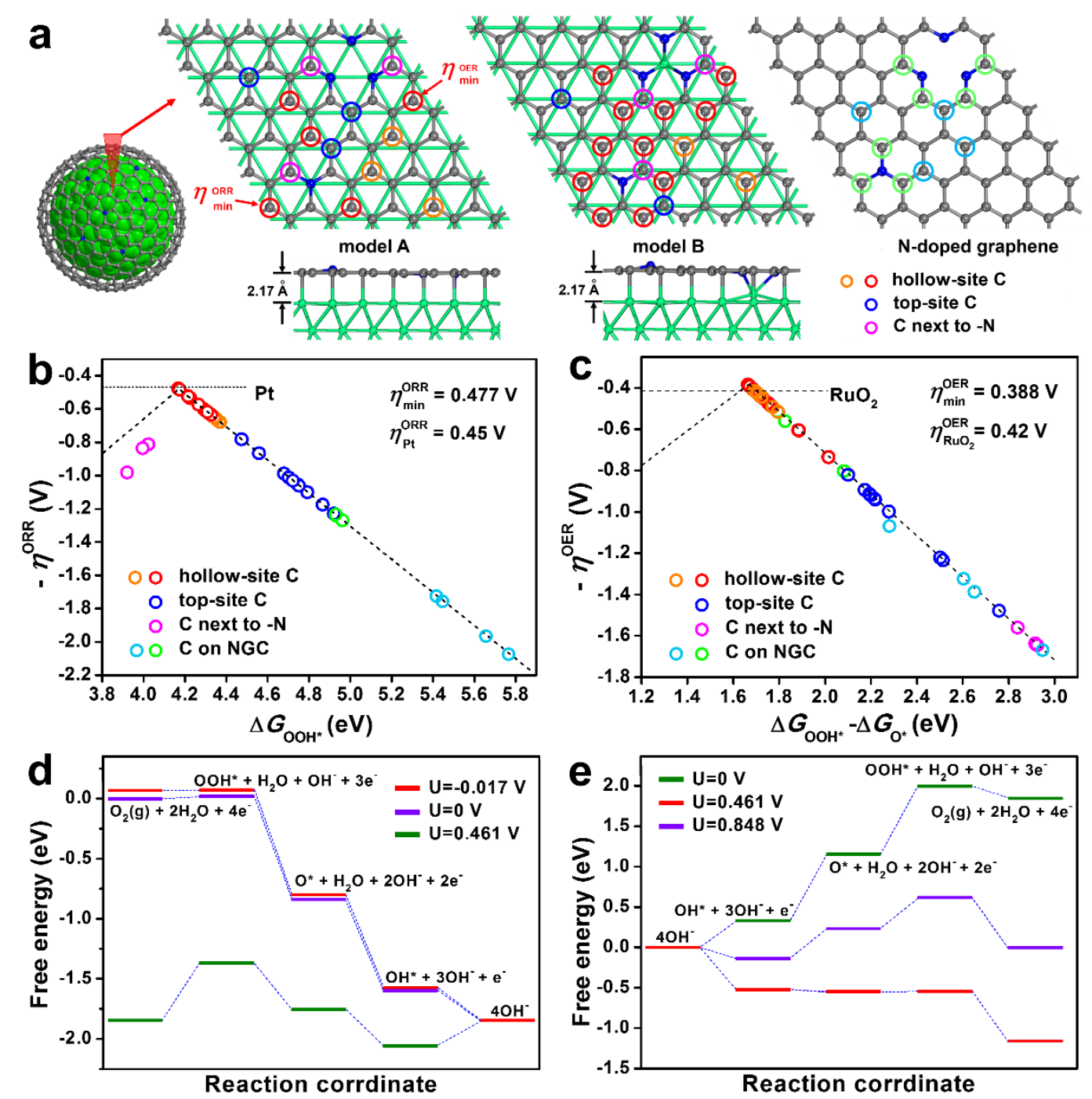

Figure 6. (a) Schematic illustration of the models of Co-NGC and N-doped graphene for DFT calculations. The $\mathrm{Co}, \mathrm{C}$ and $\mathrm{N}$ atoms are shown in green, grey and blue color, respectively. The colored circles indicate various types of reaction sites, where the ORR and OER overpotentials are calculated and plotted in (b) and (c), respectively. (b) ORR and (c) OER volcano plots of overpotential $(\eta)$ versus adsorption free energy of $\mathrm{OOH}^{*}$ and the difference between the adsorption free energy $\mathrm{OOH}^{*}$ and $\mathrm{O}^{*}$, respectively. (d, e) Free energy diagram for ORR (d) and OER (e) pathways, respectively, on Co-NGC in alkaline medium. 


\section{The table of contents}

A new strategy is developed for constructing hollow nanostructured bifunctional ORR/OER electrocatalyst with integrated high activity and fast kinetics by surface-stabilized heterogeneous contraction of core-shelled metal-organic frameworks. The resultant doubleshelled hybrid nanocages with outer shell of mesoporous Co-N-doped graphitic carbon and inner shell of microporous $\mathrm{N}$-doped carbon exhibit superior electrocatalytic performance to noble metals for oxygen reduction and evolution reactions.

Keyword: oxygen electrocatalysis, bifunctional electrocatalyst, metal-organic frameworks, hollow nanostructure, first-principles calculation

Shaohong Liu, Zhiyu Wang, Si Zhou, Fengjiao Yu, Mengzhou Yu, Chang-Yang Chiang, Wuzong Zhou, Jijun Zhao* and Jieshan Qiu ${ }^{a, *}$

\section{Metal-organic framework-derived hybrid carbon nanocages as bifunctional} electrocatalyst for oxygen reduction and evolution

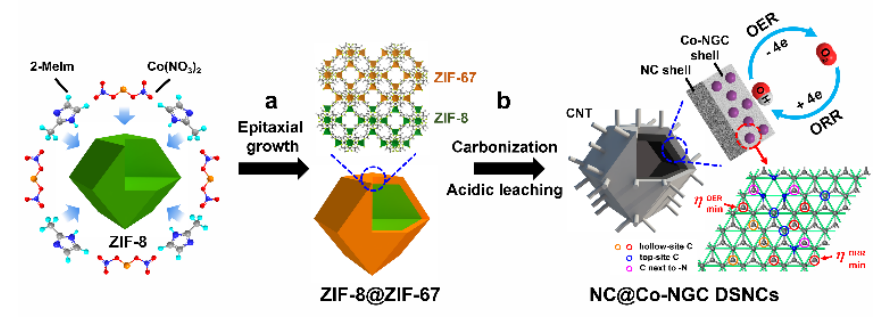


Copyright WILEY-VCH Verlag GmbH \& Co. KGaA, 69469 Weinheim, Germany, 2016.

\section{Supporting Information}

\section{Metal-organic framework-derived hybrid carbon nanocages as bifunctional electrocatalyst for oxygen reduction and evolution}

Shaohong Liu, ${ }^{a}{ }^{\dagger}$ Zhiyu Wang, ${ }^{a}{ }^{\dagger}$ Si Zhou, ${ }^{b, \dagger}$ Fengjiao Yu, ${ }^{c}$ Mengzhou Yu, ${ }^{a}$ Chang-Yang Chiang, ${ }^{c}$ Wuzong Zhou, ${ }^{c}$ Jijun Zhao ${ }^{b, *}$ and Jieshan Qiu ${ }^{a, *}$

Dr. S. H. Liu, Prof. Z. Y. Wang, Dr. S. Zhou, F. J. Yu, M. Z. Yu, C. Chiang, Prof. W. Z. Zhou, Prof. J. J. Zhao, Prof. J. S. Qiu

${ }^{a}$ State Key Lab of Fine Chemicals, Carbon Research Laboratory, Liaoning Key Lab for Energy Materials and Chemical Engineering, School of Chemical Engineering, Dalian University of Technology, Dalian 116024, China.

${ }^{b}$ Education Ministry Key Lab of Materials Modification by Laser, Ion and Electron Beams, Dalian University of Technology, Dalian 116024, China

${ }^{\mathrm{c}}$ School of Chemistry, University of St Andrews, St Andrews, KY16 9ST, United Kingdom

${ }^{\dagger}$ These authors contribute equally to this work.

E-mail: jqiu@dlut.edu.cn, zhaojj@dlut.edu.cn

\section{Experimental section}

Synthesis of ZIF-8 and ZIF-67 particles. For the synthesis of ZIF-8, two solutions were first prepared by dissolving $3 \mathrm{mmol}$ of $\mathrm{Zn}\left(\mathrm{NO}_{3}\right)_{2} \cdot 6 \mathrm{H}_{2} \mathrm{O}$ in $30 \mathrm{~mL}$ of methanol and $12 \mathrm{mmol}$ of 2methylimidazole (2-MeIm) in $10 \mathrm{~mL}$ of methanol, respectively. Then, the solution of 2-MeIm was quickly poured into the solution of $\mathrm{Zn}\left(\mathrm{NO}_{3}\right)_{2} \cdot 6 \mathrm{H}_{2} \mathrm{O}$, leading to a mixed solution that was aged for $24 \mathrm{~h}$ at room temperature. The ZIF- 8 crystals with an average edge size of around 800 , 500,300 and $150 \mathrm{~nm}$ could be yielded by using 5, 10, 20 and $30 \mathrm{~mL}$ of methanol for dissolving 2-MeIm. The as-obtained precipitates were collected by centrifugation and dried at $60^{\circ} \mathrm{C}$. ZIF67 was synthesized with the same procedure of ZIF-8 except for the use of $\mathrm{Co}\left(\mathrm{NO}_{3}\right)_{2} \cdot 6 \mathrm{H}_{2} \mathrm{O}$ as metal precursor.

Synthesis of ZIF-8@ZIF-67 core-shell structures. In a typical run, $100 \mathrm{mg}$ of ZIF-8 polyhedrons with various sizes were dispersed in $30 \mathrm{~mL}$ of methanol. Then $150 \mathrm{mg}$ of $\mathrm{Co}\left(\mathrm{NO}_{3}\right)_{2} \cdot 6 \mathrm{H}_{2} \mathrm{O}$ and a methanol solution of 2-MeIm $\left(10 \mathrm{~mL}, 32.8 \mathrm{mg} \mathrm{mL}^{-1}\right)$ were added into the suspension of ZIF-8 polyhedrons under stirring. After stirring for 24 h, the ZIF-8@ZIF-67 core-shell particles were harvested by several centrifugation-rinsing cycles with methanol, followed by drying at $60{ }^{\circ} \mathrm{C}$. For comparison, core-shelled ZIF-67@ZIF-8 particles could be also yielded by similar approach except using $\mathrm{ZIF}-67$ as the seeds and $\mathrm{Zn}\left(\mathrm{NO}_{3}\right)_{2} \cdot 6 \mathrm{H}_{2} \mathrm{O}$ for the growth of ZIF-8 shell with different thickness.

Synthesis of NC@Co-NGC DSNCs. The as-prepared ZIF-8@ZIF-67 particles were annealed at $800{ }^{\circ} \mathrm{C}$ for $5 \mathrm{~h}$ at a ramp rate of $2{ }^{\circ} \mathrm{C} \mathrm{min}^{-1}$ in flowing $\mathrm{N}_{2}$ in a tube furnace, yielding black powders that were washed in $0.5 \mathrm{M} \mathrm{H}_{2} \mathrm{SO}_{4}$ solution at $80{ }^{\circ} \mathrm{C}$ for $12 \mathrm{~h}$ to remove the metal species. The control experiments were also performed by annealing the ZIF-8@ZIF-67 particles in flowing $\mathrm{N}_{2}$ at 500, 550 and $600{ }^{\circ} \mathrm{C}$, respectively. With ZIF-8, ZIF-67 and ZIF-67@ZIF-8 as the precursors, NC, Co-NGC and Co-NGC@NC particles were prepared under identical conditions for the comparison, respectively. ${ }^{24}$ 
Material characterization. Field-emission SEM (FESEM) analysis was conducted on a JEOL JSM-6700F microscopy operated at $5 \mathrm{kV}$. Transmission electron microscopy (TEM) observation was conducted on a JEOL JEM-2011 microscopy operated at $200 \mathrm{kV}$, equipped with an Oxford Link ISIS system for elemental mapping. Powder X-ray diffraction (XRD) was performed on a PANalytical Empyrean diffractometer with $\mathrm{Cu}-\mathrm{K} \alpha$ radiation. The surface characteristics of the samples were investigated using a Thermo ESCALAB 250 X-ray photoelectron spectrometer (XPS). The textural properties of the samples were measured by Micrometrics ASAP 2020 Surface Area and Porosity Analyzer at 77 K. TGA was carried out by a NETZSCH scientific instrument in $\mathrm{N}_{2}$, heating from room temperature to $800{ }^{\circ} \mathrm{C}$ at a ramp rate of $10{ }^{\circ} \mathrm{C} \mathrm{min}^{-1}$.

Electrochemical measurement. All electrocatalytic measurements were performed using a CHI 760D electrochemical workstation with a three-electrode system at room temperature. A $0.1 \mathrm{M} \mathrm{KOH}$ aqueous solution saturated with $\mathrm{O}_{2}$ was used as the electrolyte unless otherwise stated. A glassy-carbon rotating disk electrode (RDE) or a rotating ring-disk electrode (PINE) was used as working electrode while a $\mathrm{Pt}$ foil and an $\mathrm{Ag} / \mathrm{AgCl}$ electrode saturated with $\mathrm{KCl}$ solution was employed as counter and reference electrode, respectively. All potentials measured against an $\mathrm{Ag} / \mathrm{AgCl}$ electrode in this study, was converted to potential versus RHE according to $E_{\mathrm{vs} \mathrm{RHE}}=E_{\mathrm{vs} \mathrm{Ag} / \mathrm{AgCl}}+0.059 \mathrm{pH}+0.197$. To prepare the working electrode, $4 \mathrm{mg}$ of the sample was ultrasonically dispersed in a mixture of ethanol $(0.5 \mathrm{~mL})$, deionized water $(0.485 \mathrm{~mL})$ and Nafion $(0.015 \mathrm{~mL}, 5 \mathrm{wt} . \%)$ to form a uniform suspension. A part of the catalyst ink was then loaded onto a glassy-carbon electrode by microsyringe with a mass loading of $0.4 \mathrm{mg} \mathrm{cm}^{-2}$ for all samples including commercial $\mathrm{Pt} / \mathrm{C}\left(20 \mathrm{wt} \%\right.$, Johnson Matthey) and $\mathrm{RuO}_{2}$ (Sigma-Aldrich). The polarization curves for ORR were recorded at different rotating rate from 400 to $2000 \mathrm{rpm}$ with a scan rate of $10 \mathrm{mV} \mathrm{s}^{-1}$. Methanol tolerance of the catalysts was tested by conducting the ORR in the presence of methanol $(14 \mathrm{~mL}, 3 \mathrm{M})$ in $\mathrm{O}_{2}$-saturated $\mathrm{KOH}$ solution $(100 \mathrm{~mL}, 0.1 \mathrm{M})$ by the similar procedure. For the OER test, the polarization curves were obtained at a rotating rate of $1600 \mathrm{rpm}$ with a scan rate of $10 \mathrm{mV} \mathrm{s}^{-1}$, corrected by iR-compensation. The electrical double layer capacitance $\left(C_{\mathrm{dl}}\right)$ of the samples was measured from double-layer charging curves using cyclic voltammograms $(\mathrm{CVs})$ in a small potential range of $1.10-1.15 \mathrm{~V}$ in $0.1 \mathrm{M} \mathrm{KOH}$ at the scan rate of 5, 10, 20, 30 and $40 \mathrm{mV} \mathrm{s}^{-1}$. The plot of the current density (at $1.14 \mathrm{~V}$ ) against the scan rate has a linear relationship and its slope is the $C_{\mathrm{dl}}$ of the tested catalyst.

The overall electron transfer numbers per oxygen molecule involved in a typical ORR process can be calculated from the slopes of Koutecky-Levich (K-L) plots using the following equation:

$$
1 / j=1 / j_{k}+1 / B \omega^{1 / 2}
$$

where $j_{k}$ is the kinetic current at a constant potential and $\omega$ represents the electrode rotating speed. $B$ can be determined from the slope of the K-L plots based on Levich equation:

$$
B=0.2 n F\left(D_{2}\right)^{2 / 3} v^{-1 / 6} \mathrm{Co}_{2}
$$

where $n$ represents the transferred electron number per oxygen molecule, $F$ is Faraday constant (96485 $\left.\mathrm{C} \mathrm{mol}^{-1}\right), \mathrm{Do}_{2}$ is the diffusion coefficient of $\mathrm{O}_{2}\left(1.9 \times 10^{-5} \mathrm{~cm}^{2} \mathrm{~s}^{-1}\right), v$ is the kinetic viscosity $\left(v=0.01 \mathrm{~cm}^{2} \mathrm{~s}^{-1}\right)$ and $\mathrm{Co}_{2}$ is the bulk concentration of $\mathrm{O}_{2}\left(1.2 \times 10^{-6} \mathrm{~mol} \mathrm{~cm}^{-3}\right)$. When expressing the rotation speed in rpm, the constant 0.2 is applied.

In Tafel plot, the kinetic current was calculated from the mass-transport correction of RDE data by the followed equation:

$$
j_{k}=\left(j \times j_{L}\right) /\left(j_{L}-j\right)
$$


where $j$ is the current density and $j_{L}$ is the limiting current density.

RRDE technique was also employed to determine the reaction pathway (electron transfer number) for ORR by detecting the $\mathrm{HO}_{2}^{-}$formation, in which the ring potential was held constantly at $1.50 \mathrm{~V}$ vs. RHE. The disk electrode was scanned at a rate of $10 \mathrm{mV} \mathrm{s}^{-1}$ with a rotation rate of $1600 \mathrm{rpm}$. The electron transfer number $(n)$ was determined by the followed equation:

$$
n=4 I_{d} /\left(I_{d}+I_{r} / N\right)
$$

where $I_{d}$ is disk current, $I_{r}$ is ring current, and $N$ is the current collection efficiency.

Zn-air battery test. The primary Zn-air batteries were tested in CR2032 coin-type cells with a polished $\mathrm{Zn}$ plate as the anode, a cellulose film as the separator and $6 \mathrm{M} \mathrm{KOH}$ solution as the electrolyte. The air cathodes were prepared by casting a mixture of catalyst (NC@Co-NGC DSNC, Pt/C or $\mathrm{RuO}_{2}$ ), Nafion (5 wt.\%) and ethanol onto a gas diffusion layer (TGP-H-060, Torray), followed by vacuum drying at $80{ }^{\circ} \mathrm{C}$ for $12 \mathrm{~h}$. The rechargeable $\mathrm{Zn}$-air batteries were tested using identical cell configuration except add $\mathrm{Zn}(\mathrm{Ac})_{2}(0.2 \mathrm{M})$ into $6 \mathrm{M} \mathrm{KOH}$ electrolyte. The average catalyst loading was $0.5 \mathrm{mg} \mathrm{cm}$. Battery tests were performed on a LAND CT2001A tester in air. Galvanostatic discharge and charge cycling was conducted by a recurrent galvanic pulse method at a constant current density of $10 \mathrm{~mA} \mathrm{~cm}^{-2}$ with each cycle for $10 \mathrm{~min}$. Polarization curves were collected at a scan rate of $10 \mathrm{mV} \mathrm{s}^{-1}$.

Computational method. Density functional theory (DFT) calculations are performed by Vienna $a b$ initio simulation package (VASP) ${ }^{1}$ using the plane wave basis set with an energy cutoff of $500 \mathrm{eV}$, the projector augmented wave (PAW) potentials ${ }^{2}$, and the generalized gradient approximation (GGA) parameterized by Perdew, Burke and Ernzerhof (PBE) for the exchangecorrelation functional ${ }^{3}$. As the radius of realistic Co-embedded $\mathrm{N}$-doped graphitic carbon structures in Co-NGC shells attains several nanometers, a slab model consisting of single layer $\mathrm{N}$-doped graphene adsorbed on (111) surface of $f c c$ Co is used to represent the Co-NGC surface. A three-layer slab model is adopted for the Co crystal, with a vacuum region of $14 \AA$ in the outof-plane direction. The in-plane lattice parameters of graphene $(2.46 \AA)$ and Co $(2.51 \AA)$ are very $\operatorname{close}^{4,5}$, and thus we expand and compress their in-plane lattice by $1 \%$, and use a lattice parameter of $2.48 \AA$ for both materials. Consequently, Co-NGC comprise of two types of C sites: on right top or hollow sites with respect to the Co lattice (denoted as the top-site $\mathrm{C}$ and hollow-site $\mathrm{C}$, respectively). To accommodate the $\mathrm{N}$ dopants in graphene, a periodic supercell with the in-plane dimension consisting of $6 \times 6$ unit cells is used for both graphene and Co. The Brillouin zones of the supercell are sampled by $2 \times 2 \times 1$ uniform k-point meshes. The model structures are optimized by only ionic and electronic degrees of freedom using thresholds for the total energy of $10^{-4} \mathrm{eV}$ and force of $0.02 \mathrm{eV} / \AA$. During the structural relaxation, the bottom layer of Co atoms is fixed to mimic a semi-infinite metal solid. The semi-empirical dispersioncorrected DFT-D3 scheme proposed by Grimme ${ }^{6}$ is used to describe the van der Waals interactions between graphene and the Co surface.

Three pyridinic-type $\mathrm{N}$ and one graphitic-type $\mathrm{N}$ atoms are uniformly substituted into the graphene lattice, such that atomic percentage of $\mathrm{N}$ with respect to $\mathrm{C}$ is about $6 \%$, close to the experimental value. Two models of Co-NGC structure are considered in terms of the location of $\mathrm{N}$ dopants: on the right top or hollow sites with respect to the Co lattice (denoted as model $\mathrm{A}$ and $\mathrm{B}$, respectively). The energy of latter is higher by $0.46 \mathrm{eV}$. The optimized interlayer distance between the N-doped graphene and the Co surface is $2.17 \AA$ for both model structures. For comparison, the model of an isolated N-doped graphitic carbon sheet (NGC) with the same scale is also constructed. 
To evaluate the ORR and OER activities, we calculate the adsorption free energy of the oxygenated intermediate species, including $\mathrm{OOH}^{*}, \mathrm{OH}^{*}$ and $\mathrm{O}^{*}$, involved in ORR and OER on different $\mathrm{C}$ sites of both models. The overpotentials of ORR and OER are then determined by the standard hydrogen electrode (SHE) method developed by Norskov et al. ${ }^{7,8,9}$.

In alkaline medium, the ORR could occur in the following 4-electron reaction pathway ${ }^{10,11}$ :

$$
\begin{array}{r}
*+\mathrm{O}_{2}(\mathrm{~g})+\mathrm{H}_{2} \mathrm{O}(\mathrm{l})+\mathrm{e}^{-} \rightarrow \mathrm{OOH}^{*}+\mathrm{OH}^{-} \\
\mathrm{OOH}^{*}+\mathrm{e}^{-} \rightarrow \mathrm{O}^{*}+\mathrm{OH}^{-} \\
\mathrm{O}^{*}+\mathrm{H}_{2} \mathrm{O}(\mathrm{l})+\mathrm{e}^{-} \rightarrow \mathrm{OH}^{*}+\mathrm{OH}^{-} \\
\mathrm{OH}^{*}+\mathrm{e}^{-} \rightarrow *+\mathrm{OH}^{-}
\end{array}
$$

where * represents an active site on the graphene surface.

In alkaline medium, the OER could occur via a 4-electron reaction pathway:

$$
\begin{gathered}
*+\mathrm{OH}^{-} \rightarrow \mathrm{OH}^{*}+\mathrm{e}^{-} \\
\mathrm{OH}^{*}+\mathrm{OH}^{-} \rightarrow \mathrm{O}^{*}+\mathrm{H}_{2} \mathrm{O}(\mathrm{l})+\mathrm{e}^{-} \\
\mathrm{O}^{*}+\mathrm{OH}^{-} \rightarrow \mathrm{OOH}^{*}+\mathrm{e}^{-} \\
\mathrm{OOH}^{*}+\mathrm{OH}^{-} \rightarrow *+\mathrm{O}_{2}(\mathrm{~g})+\mathrm{H}_{2} \mathrm{O}(\mathrm{l})+\mathrm{e}^{-}
\end{gathered}
$$

The adsorption energies $\left(\Delta E_{\mathrm{ads}}\right)$ of $\mathrm{OOH}^{*}, \mathrm{OH}^{*}$ and $\mathrm{O}^{*}$ are calculated by referring their DFT total energy to that of a clean Co-NGC surface, and $\mathrm{H}_{2} \mathrm{O}$ and $\mathrm{H}_{2}$ in the gas phase. The adsorption free energy $\left(\Delta G_{\text {ads }}\right)$ is obtained by

$$
\Delta G_{\mathrm{ads}}=\Delta E_{\mathrm{ads}}+\Delta \mathrm{ZPE}-T \Delta S
$$

where $\triangle \mathrm{ZPE}$ and $\Delta S$ are the contributions to the free energy from the zero-point vibration energy and entropy, respectively ${ }^{12}$. Only the vibration entropy contribution is considered here. The calculation of vibrational frequencies of the $\mathrm{OOH}^{*}, \mathrm{OH}^{*}$ and $\mathrm{O}^{*}$ on the Co-NGC surface gives $\triangle \mathrm{ZPE}-T \Delta S=0.32,0.30$ and $0.05 \mathrm{eV}$, respectively $(T=298 \mathrm{~K})$. It is in good agreement with the previous theoretical results ${ }^{7,8}$. Entropy contributions of $-0.14 \mathrm{eV}$ and $-0.11 \mathrm{eV}$ are used for gaseous $\mathrm{H}_{2}$ at the standard condition and for gaseous $\mathrm{H}_{2} \mathrm{O}$ at the equilibrium pressure of 0.035 bars in contact with liquid water at $298 \mathrm{~K}$, respectively ${ }^{7}, 13$. Supplementary Fig. 19 shows the $\Delta G_{\text {ads }}$ of the oxygenated species on different $\mathrm{C}$ sites in the model $\mathrm{A}$ and $\mathrm{B}$, compared with that of an isolated NGC layer. The adsorption free energy of $\mathrm{OOH}^{*}, \mathrm{OH}^{*}$ and $\mathrm{O}^{*}$ are linearly correlated, and in good accordance with the previous studies ${ }^{8,14}$.

For each reaction step in ORR/OER, the Gibbs free energy of formation is given by

$$
\Delta G=\Delta E_{\mathrm{DFT}}+\Delta \mathrm{ZPE}-T \Delta S-e U
$$

where $\Delta E_{\mathrm{DFT}}, \Delta \mathrm{ZPE}$ and $\Delta S$ are the change of DFT total energy, zero-point energy, and entropy from the initial to the final state; $U$ is the electrode potential and $e$ is the charge transferred. The reference potential is set to be that of the standard hydrogen electrode ${ }^{7,9}$. The overpotential of ORR is then determined by ${ }^{7}$

$$
\eta^{\mathrm{ORR}}=\Delta G_{\max } / e+0.461 \mathrm{~V}
$$

where $\Delta G_{\max }$ is the maximum Gibbs free energy of formation among the four reaction steps; $0.461 \mathrm{~V}$ is the equilibrium potential at the standard condition and in an alkaline environment with $\mathrm{pH}=13^{8,14}$. At the equilibrium potential, the overall reaction $\mathrm{O}_{2}(\mathrm{~g})+\mathrm{H}_{2} \mathrm{O}(\mathrm{l})+4 \mathrm{e}^{-} \rightarrow$ $4 \mathrm{OH}^{-}$has a zero Gibbs free energy of formation. For OER, the overpotential is then determined by 


$$
\eta^{\mathrm{OER}}=\Delta G_{\mathrm{max}} / e-0.461 \mathrm{~V}
$$

Based on the calculated adsorption free energies of various oxygenated intermediate species, the Gibbs free energy of formation for each reaction step can be obtained, and the overpotentials of ORR and OER are extracted by Eq.(S15) and (S16), respectively. 

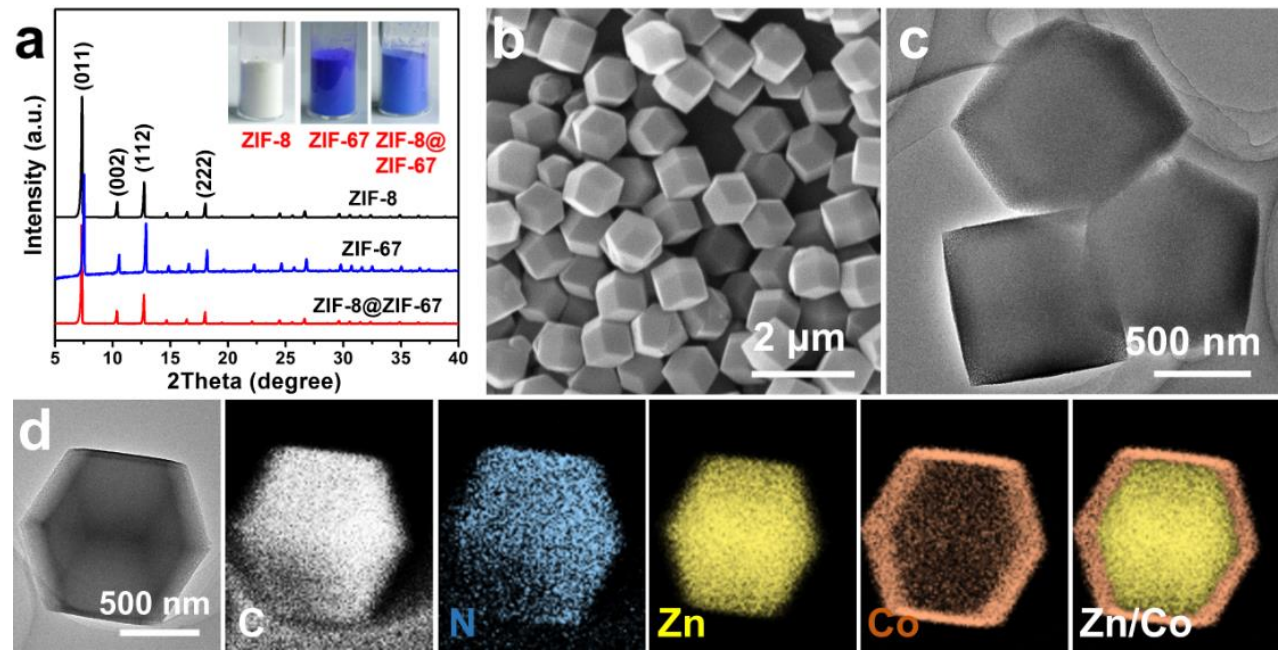

Figure S1. (a) XRD patterns of ZIF-8, ZIF-67 and core-shelled ZIF-8@ZIF-67 polyhedrons. Inset is the digital photo of the samples. (b) FESEM image and (c) TEM image of ZIF-8@ZIF67 polyhedrons. (d) Elemental mapping showing the distribution of C, N, Zn, and Co in ZIF8@ZIF-67 polyhedrons.

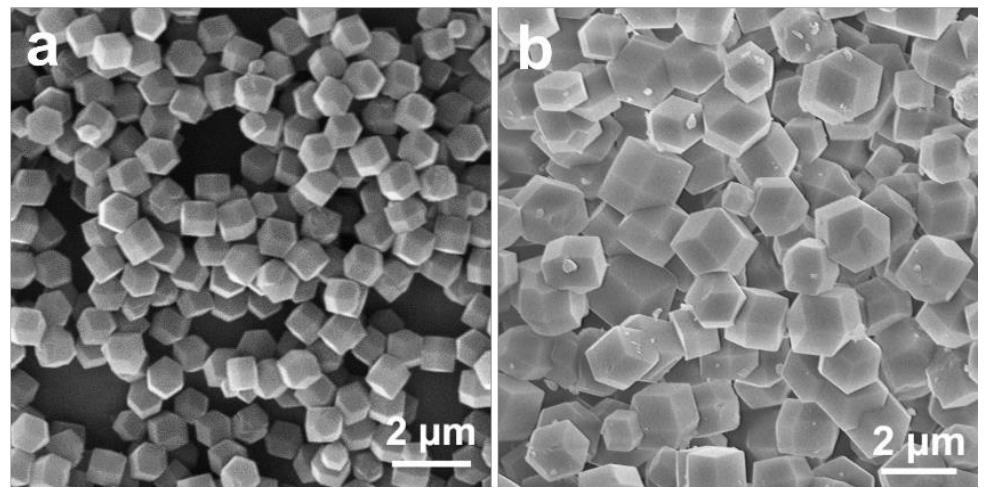

Figure S2. FESEM images of (a) ZIF-8 and (b) ZIF-67 particles.

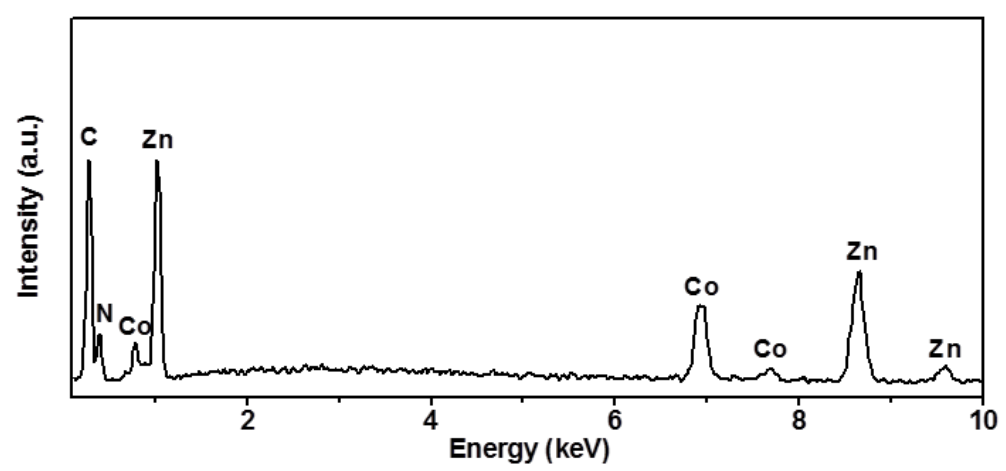

Figure S3. EDX spectrum of ZIF-8@ZIF-67 particles. 


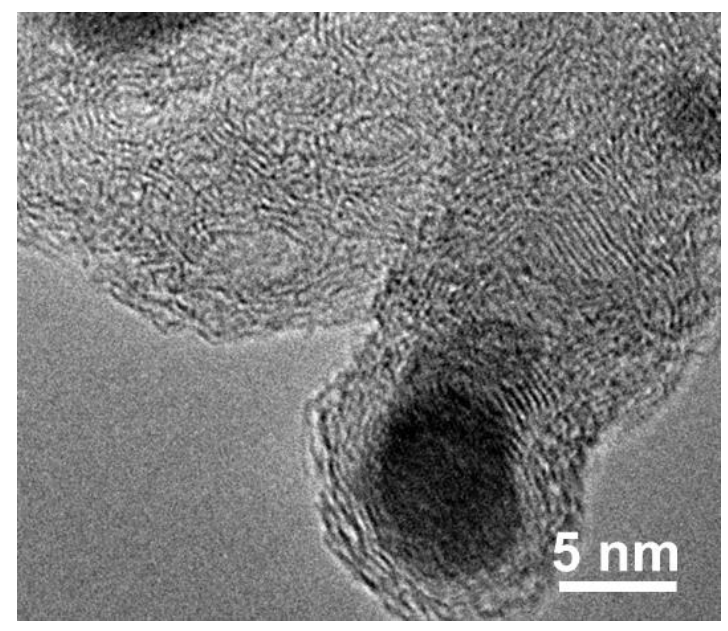

Figure S4. HRTEM image of a Co nanoparticle encapsulated in several graphitic carbon layers in Co-NGC shell.

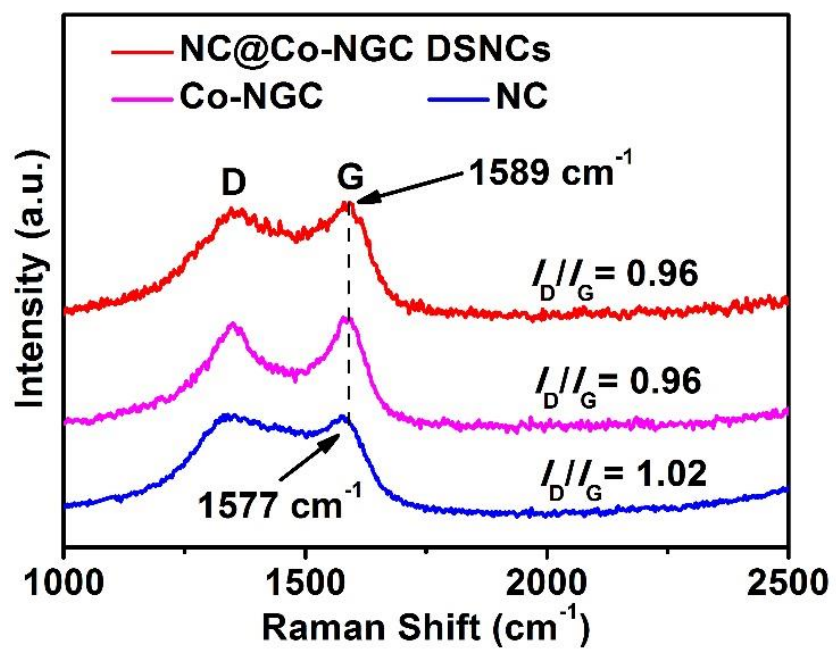

Figure S5. Raman spectra of NC@Co-NGC DSNCs, ZIF-67 derived Co-NGC and ZIF-8 derived NC.

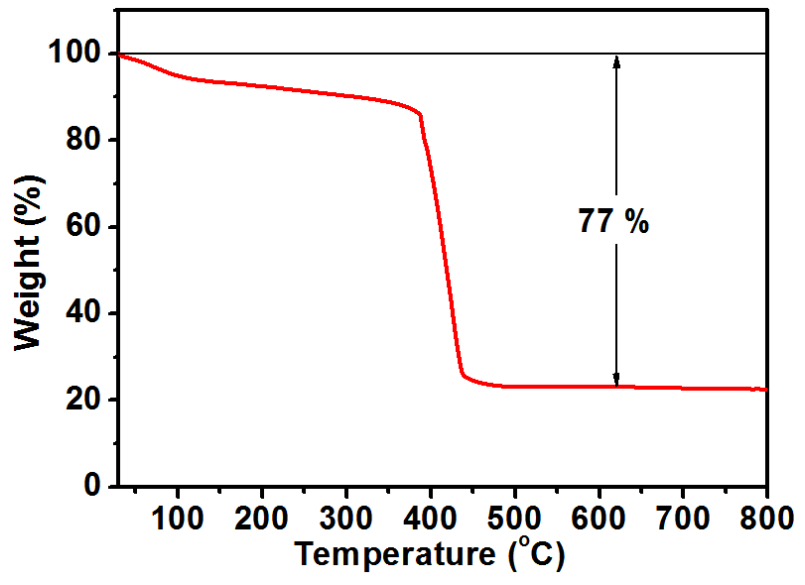

Figure S6. TGA curve of NC@Co-NGC DSNCs in flowing air at a ramp rate of $10{ }^{\circ} \mathrm{C} \mathrm{min}-1$. 

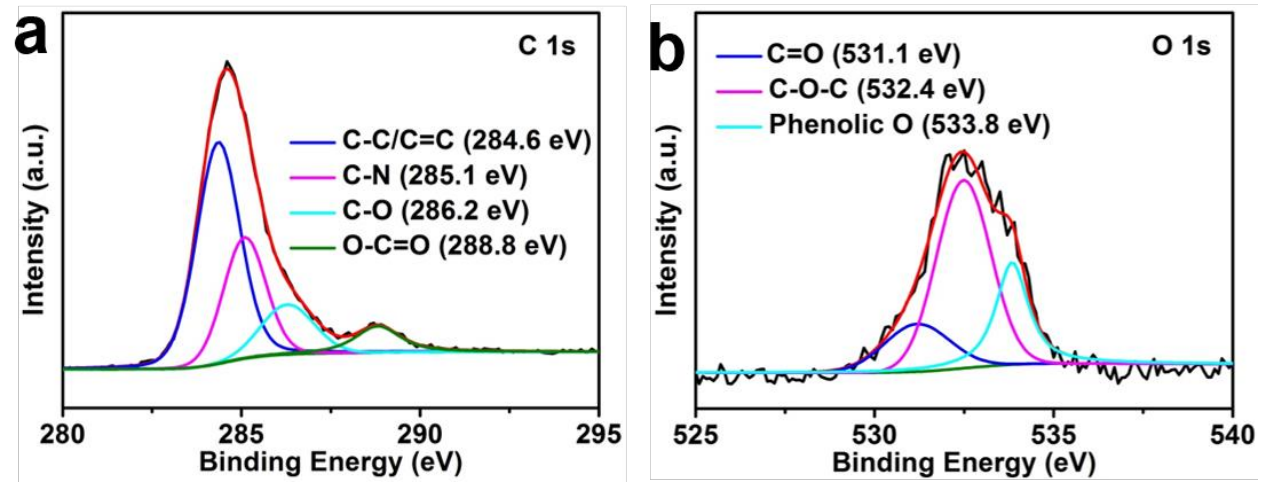

Figure S7. (a) C 1s and (b) O 1s XPS spectra of NC@Co-NGC DSNCs.

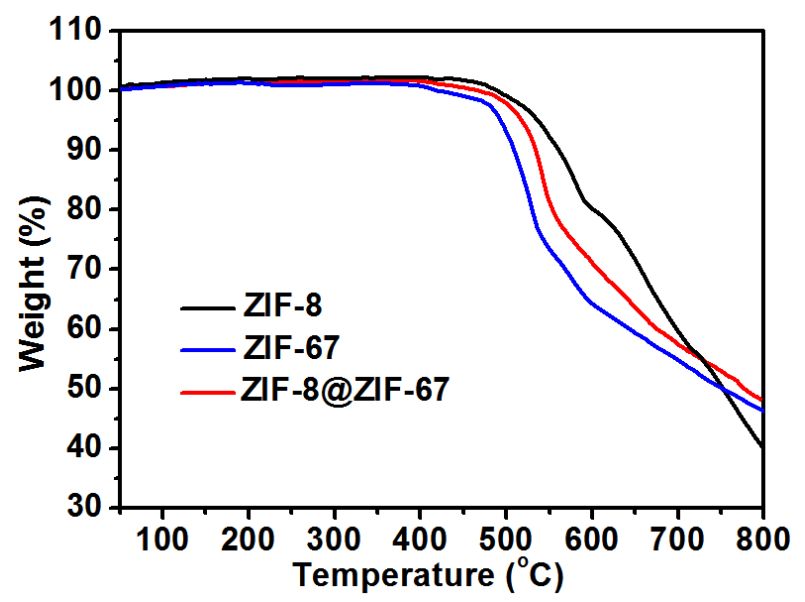

Figure S8. TGA curves of ZIF-8, ZIF-67 and ZIF-8@ZIF-67 samples in flowing $\mathrm{N}_{2}$ at a ramp rate of $10^{\circ} \mathrm{C} \mathrm{m^{-1 }}$.

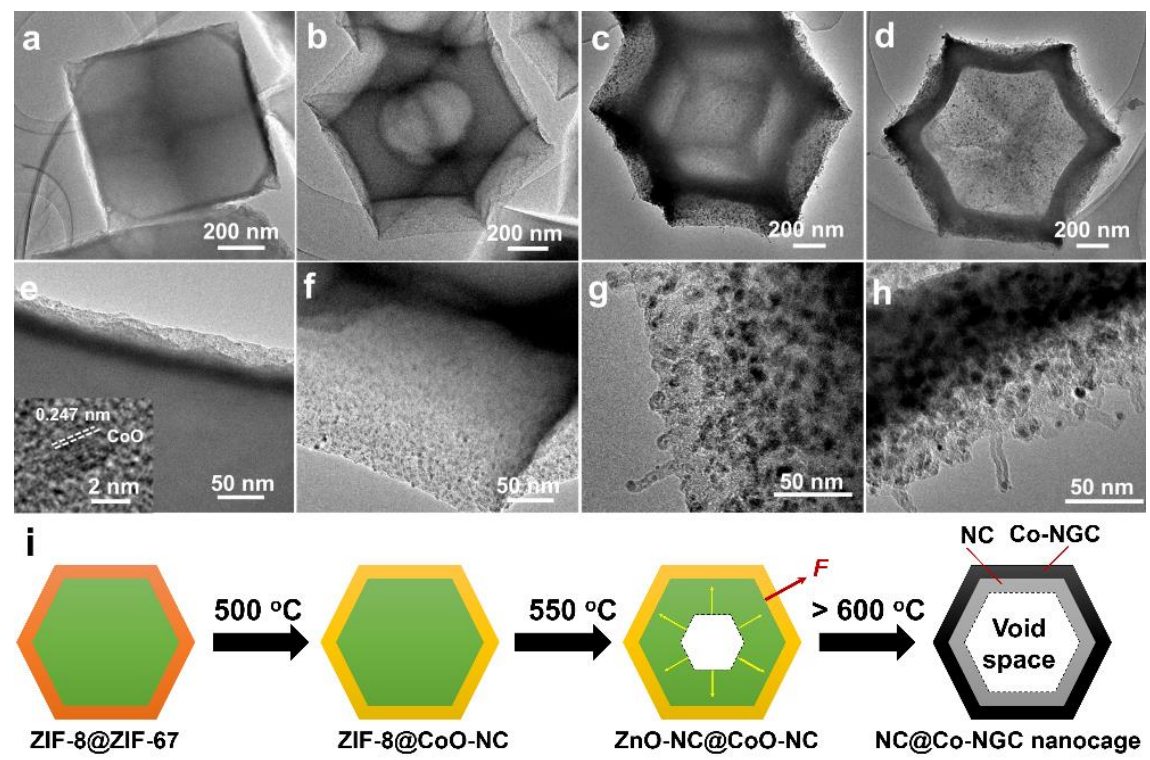

Figure S9. (a-h) TEM images of core-shell ZIF-8@ZIF-67 nanoparticles annealed at (a, e) 500 ${ }^{\circ} \mathrm{C}$, (b, f) $550{ }^{\circ} \mathrm{C},(\mathrm{c}, \mathrm{g}) 600{ }^{\circ} \mathrm{C}$, and $(\mathrm{d}, \mathrm{h}) 800{ }^{\circ} \mathrm{C}$. (i) Schematic of the formation process of NC@Co-NGC DSNCs. 


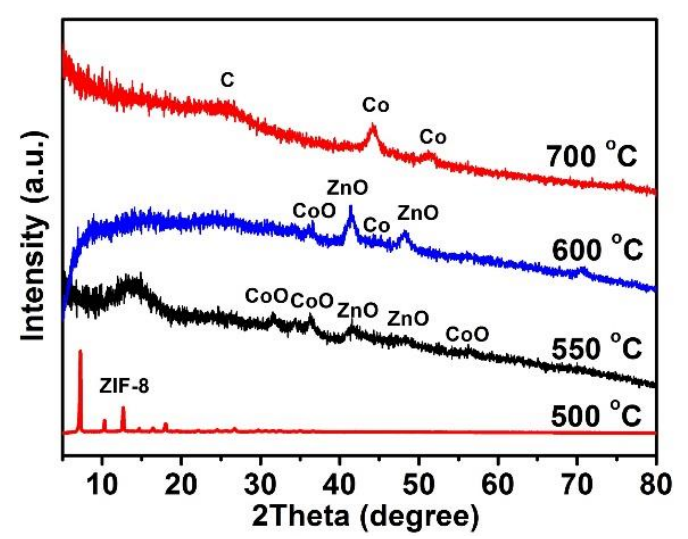

Figure S10. XRD patterns of the samples obtained by annealing core-shell ZIF-8@ZIF-67 particles at different temperatures.
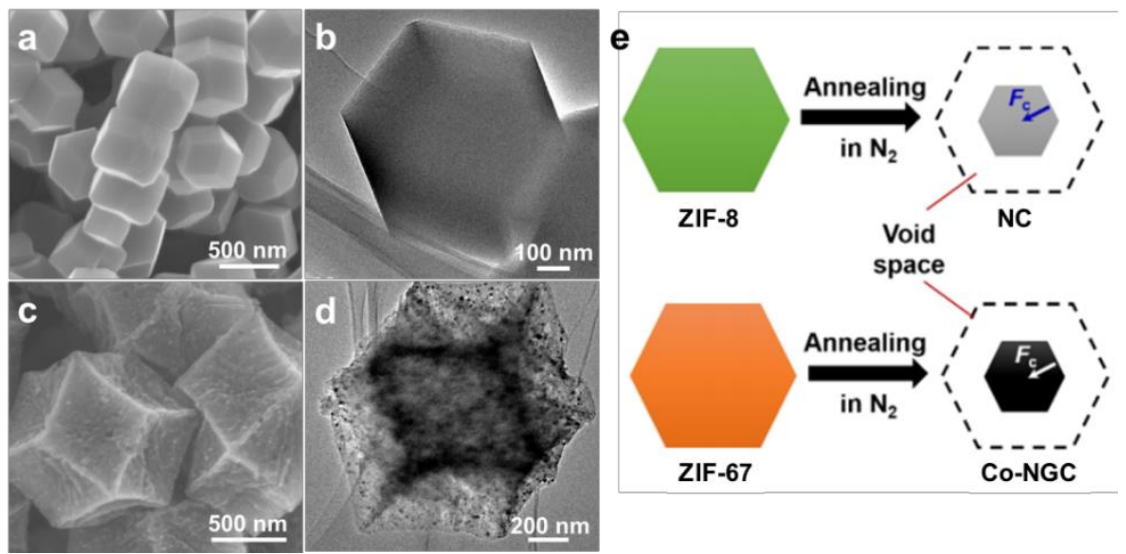

Figure S11. (a) FESEM and (b) TEM images of ZIF-8 derived NC particles. (c) FESEM and (d) TEM images of ZIF-67 derived Co-NGC particles. Both of them are obtained by annealing the parent ZIF at $800{ }^{\circ} \mathrm{C}$. (e) Schematic of the formation mechanism of ZIF-derived solid particles.

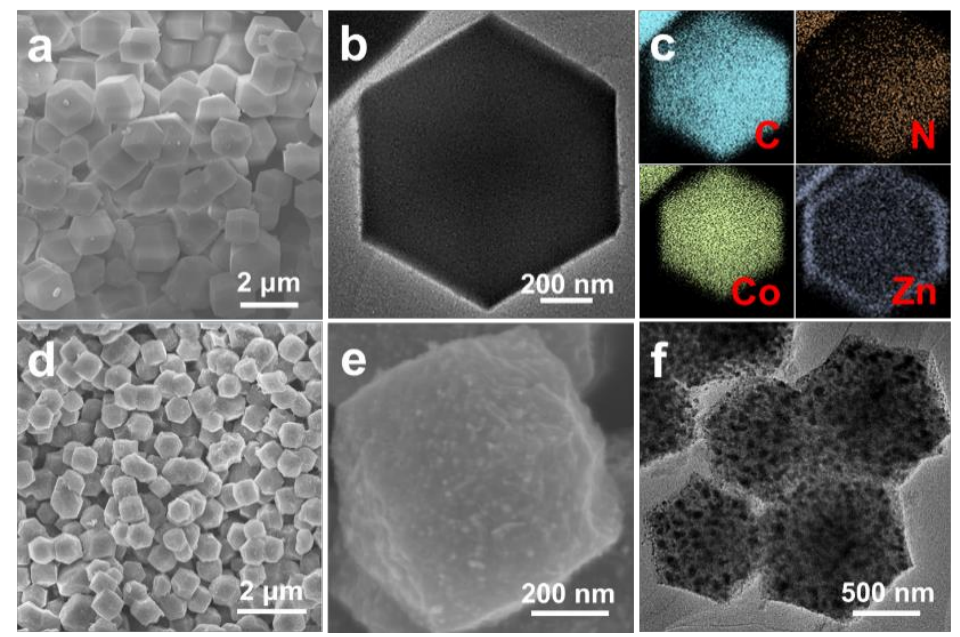

Figure S12. (a) FESEM and (b) TEM images of ZIF-67@ZIF-8 polyhedrons; (c) elemental mapping showing the presence of C, N, Co and Zn in core-shelled ZIF-67@ZIF-8 polyhedrons; (d, e) FESEM and (f) TEM images of ZIF-67@ZIF-8 derived Co-NGC@NC nanoparticles. 

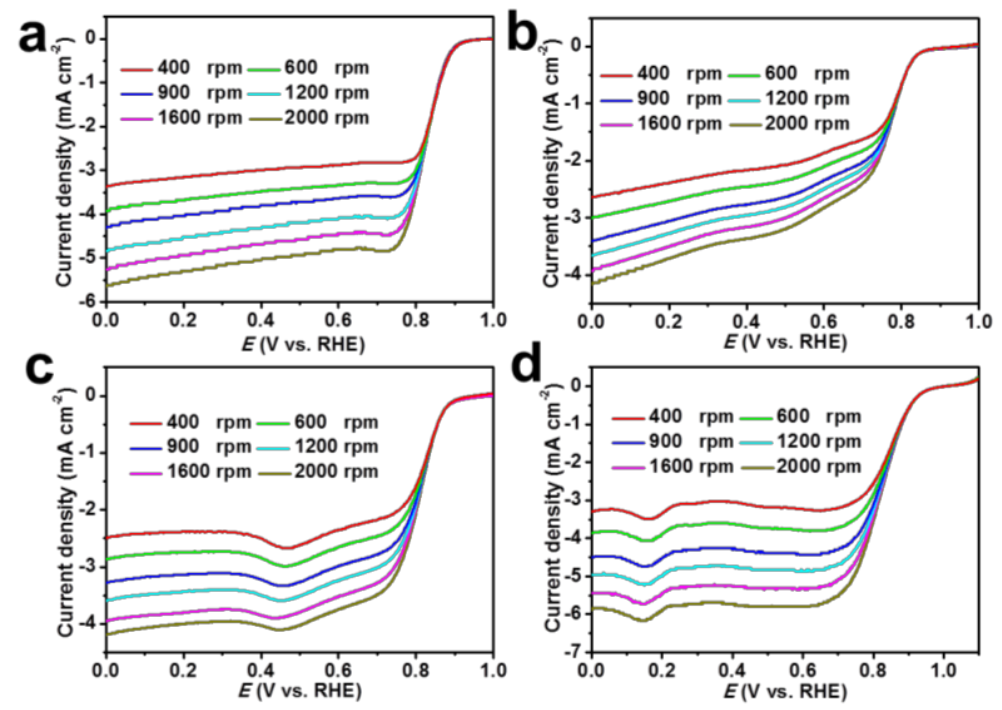

Figure S13. LSVs of (a) NC@Co-NGC DSNC, (b) NC, (c) Co-NGC and (d) Pt/C catalysts at different rotation rates in $\mathrm{O}_{2}$-saturated $0.1 \mathrm{M} \mathrm{KOH}$ solution at a scan rate of $10 \mathrm{mV} \mathrm{s}^{-1}$.
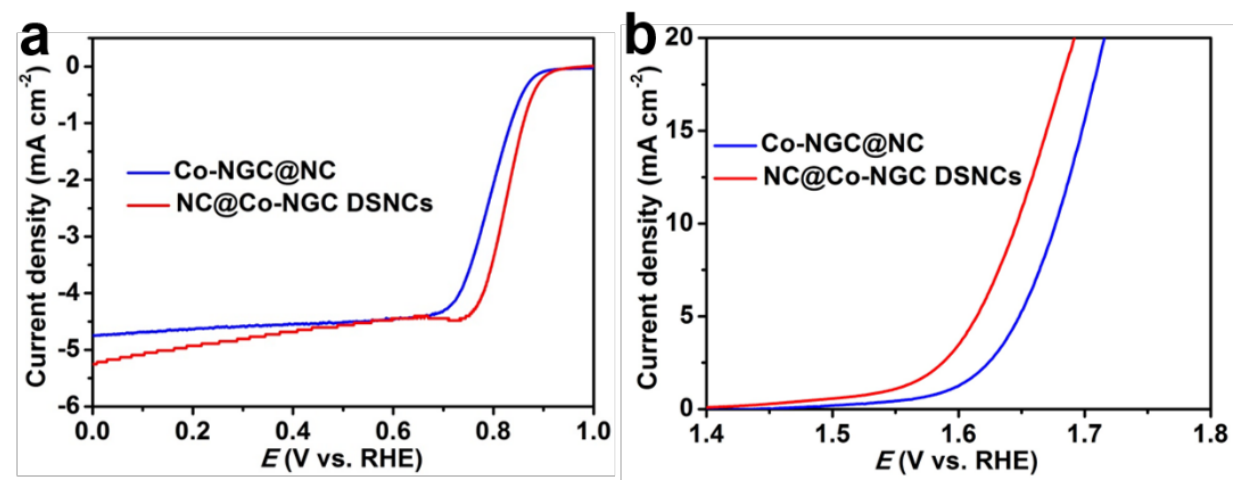

Figure S14. (a) ORR and (b) OER polarization curves of Co-NGC@NC and NC@Co-NGC DSNC catalysts, measured at a rotation rate of $1600 \mathrm{rpm}$ in $\mathrm{O}_{2}$-saturated $0.1 \mathrm{M} \mathrm{KOH}$ solution at a scan rate of $10 \mathrm{mV} \mathrm{s}^{-1}$.
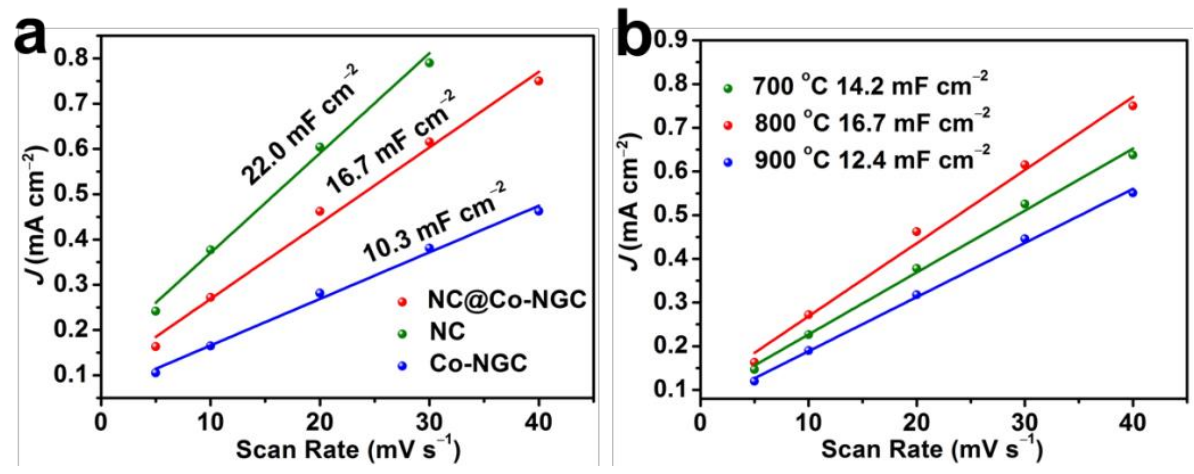

Figure S15. Plots of the current density at $1.09 \mathrm{~V}$ vs. the scan rate to determine the double layer capacitance $\left(C_{\mathrm{dl}}\right)$ of (a) the NC@Co-NGC DSNC, Co-NGC and NC samples, (b) the NC@CoNGC DSNCs obtained at different temperature. 

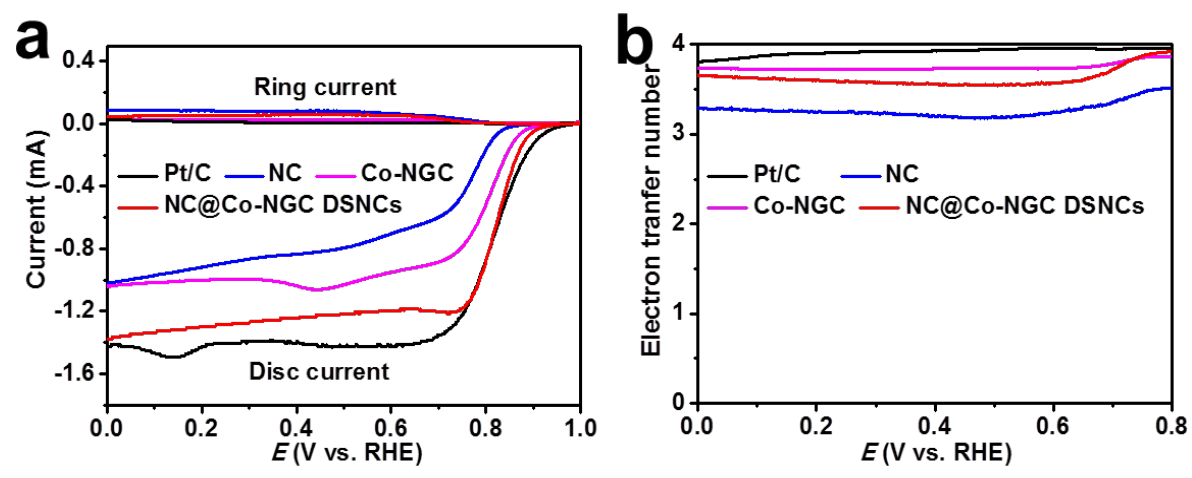

Figure S16. (a) RRDE measurements of NC, Co-NGC, NC@Co-NGC DSNC and Pt/C catalysts at a rotation rate of $1600 \mathrm{rpm}$ in $\mathrm{O}_{2}$-saturated $0.1 \mathrm{M} \mathrm{KOH}$ solution at a scan rate of 10 $\mathrm{mV} \mathrm{s}^{-1}$. (b) The electron transfer number for ORR on NC, Co-NGC, NC@Co-NGC DSNC and $\mathrm{Pt} / \mathrm{C}$ catalysts, obtained based on the disk and ring currents in RRDE measurements (ring potential: $1.50 \mathrm{~V})$.

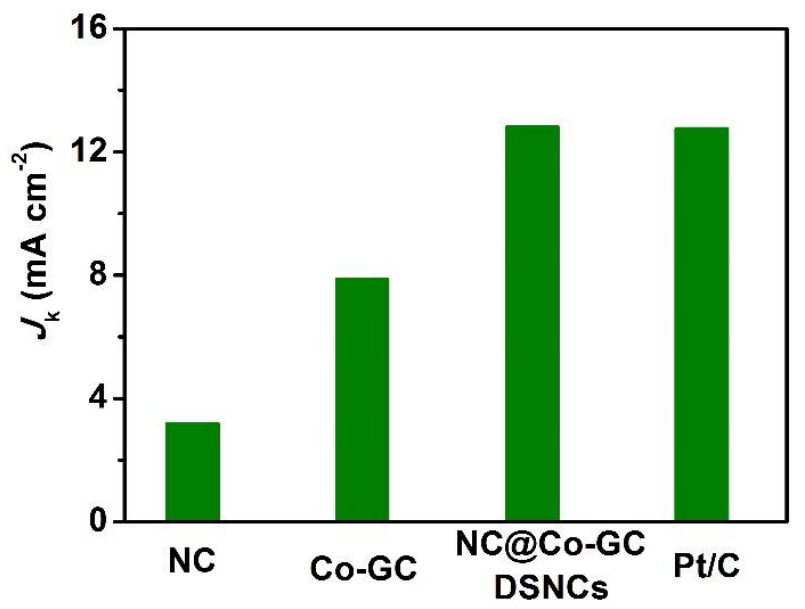

Figure S17. Kinetic currents of various samples for ORR at $0.71 \mathrm{~V}$ vs. RHE.
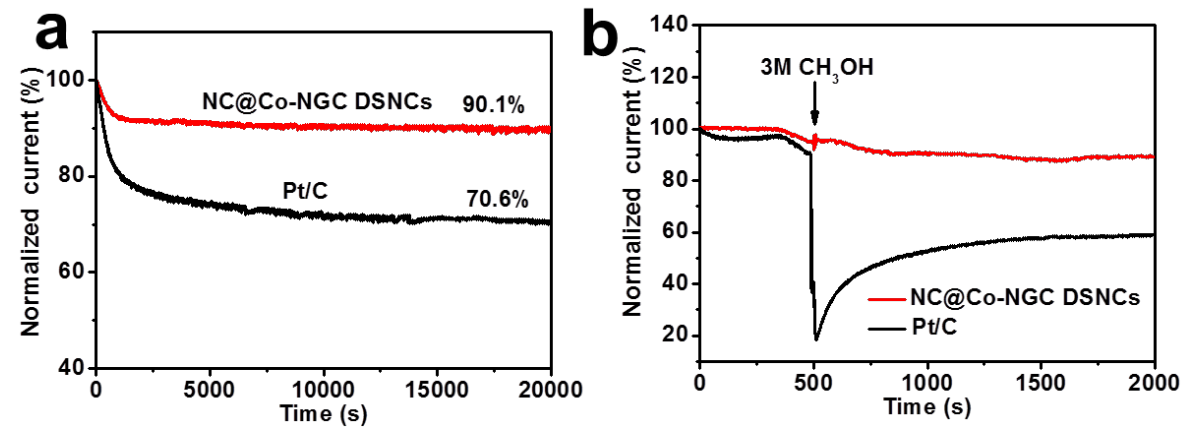

Figure S18. (a) Chronoamperometric responses of NC@Co-NGC DSNC and Pt/C catalysts at $0.56 \mathrm{~V}$ in $\mathrm{O}_{2}$-saturated $0.1 \mathrm{M} \mathrm{KOH}$ solution at $1600 \mathrm{rpm}$; (b) Chronoamperometric responses of NC@Co-NGC DSNC and Pt/C catalysts upon addition of $3 \mathrm{M}$ methanol into $\mathrm{O}_{2}$-saturated $0.1 \mathrm{M} \mathrm{KOH}$ at $0.56 \mathrm{~V}$. 

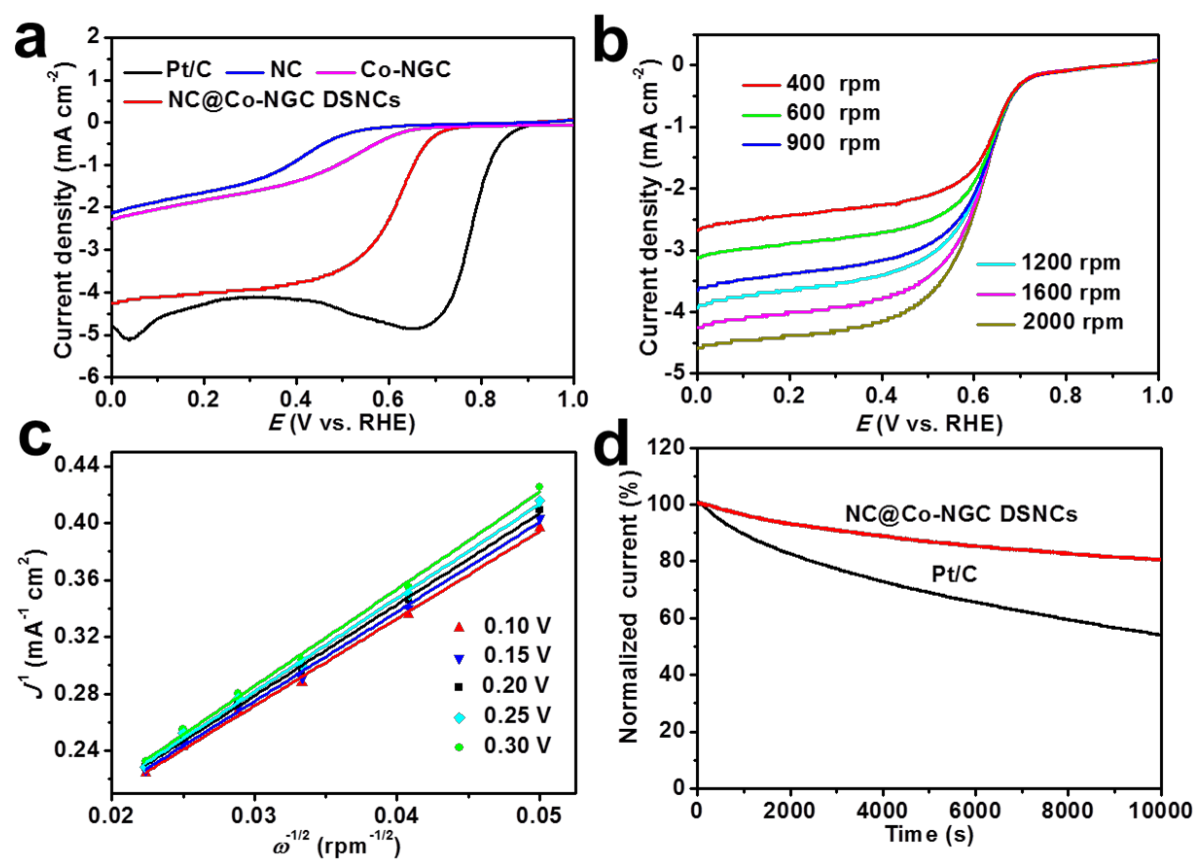

Figure S19. (a) ORR polarization curves of NC@Co-NGC DSNCs, Pt/C, Co-NGC and NC catalyst at a rotation rate of $1600 \mathrm{rpm}$ at a scan rate of $10 \mathrm{mV} \mathrm{s}^{-1}$. (b) LSVs of NC@Co-NGC DSNCs at different rotation rates at a scan rate of $10 \mathrm{mV} \mathrm{s}^{-1}$. (c) Koutecky-Levich plots of NC@Co-NGC DSNCs at various potentials. (d) Chronoamperometric responses of NC@CoNGC DSNCs and Pt/C catalyst at $0.50 \mathrm{~V}$. All the catalysts are tested in $\mathrm{O}_{2}$-saturated $0.5 \mathrm{M}$ $\mathrm{H}_{2} \mathrm{SO}_{4}$ solution, except for $\mathrm{Pt} / \mathrm{C}$ that is tested in $0.1 \mathrm{M} \mathrm{HClO}_{4}$ to avoid the catalyst poisoning caused by bisulfate.
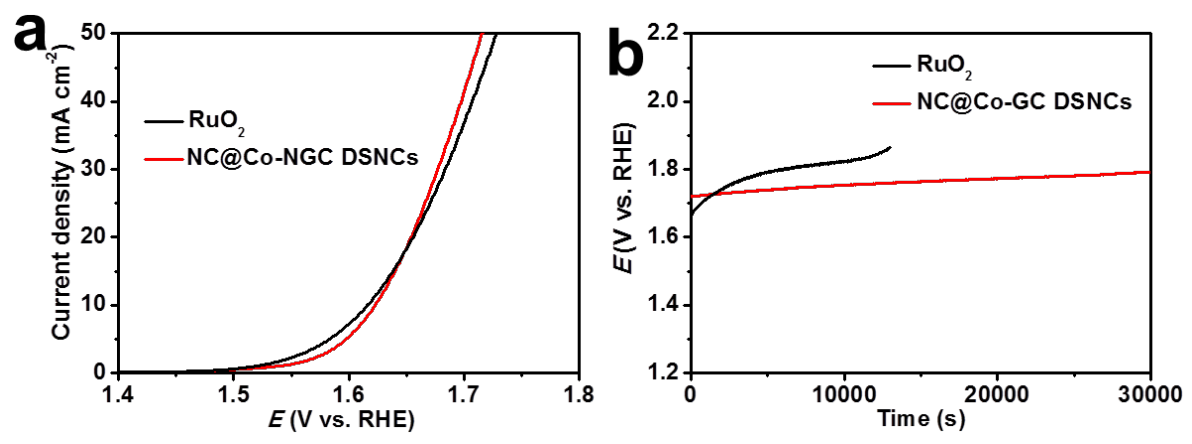

Figure S20. (a) Polarization curves of $\mathrm{RuO}_{2}$ and $\mathrm{NC} @$ Co-NGC DSNC catalysts in an $\mathrm{O}_{2}$ saturated $1 \mathrm{M} \mathrm{KOH}$ solution at a scan rate of $10 \mathrm{mV} \mathrm{s}^{-1}$. (b) Chronopotentiometric response of $\mathrm{RuO}_{2}$ and NC@Co-NGC DSNC catalysts at a constant current density of $10.0 \mathrm{~mA} \mathrm{~cm}^{-2}$ in an $\mathrm{O}_{2}$-saturated $0.1 \mathrm{M} \mathrm{KOH}$ solution. 

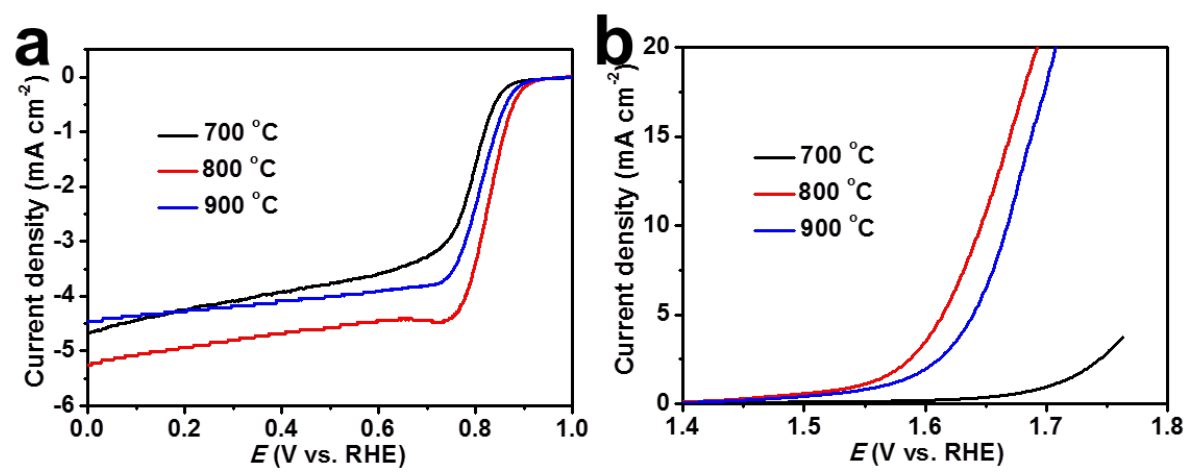

Figure S21. (a) ORR and (b) OER polarization curves of NC@Co-NGC DSNC catalysts obtained at different annealing temperatures, measured at a rotation rate of $1600 \mathrm{rpm}$ in $\mathrm{O}_{2}$ saturated $0.1 \mathrm{M} \mathrm{KOH}$ solution at a scan rate of $10 \mathrm{mV} \mathrm{s}^{-1}$.

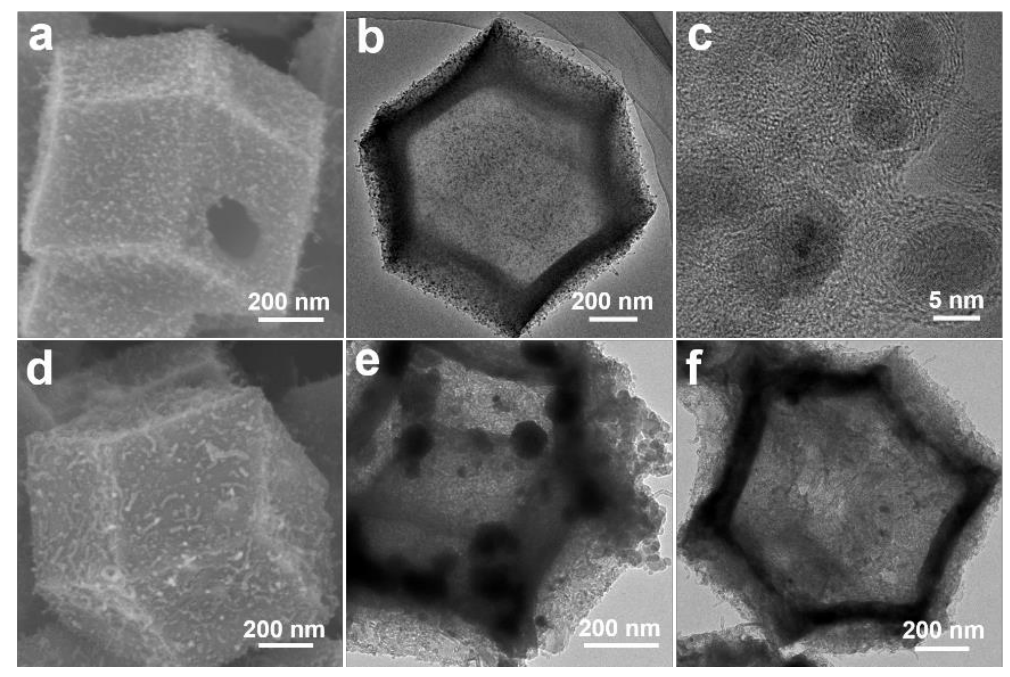

Figure S22. (a) FESEM and (b, c) TEM images of NC@Co-NGC DSNCs obtained at $700{ }^{\circ} \mathrm{C}$. (d) FESEM and (e) TEM images of NC@Co-NGC DSNCs obtained at $900{ }^{\circ} \mathrm{C}$. (f) The large Co particles in this sample can be easily removed by acidic etching.
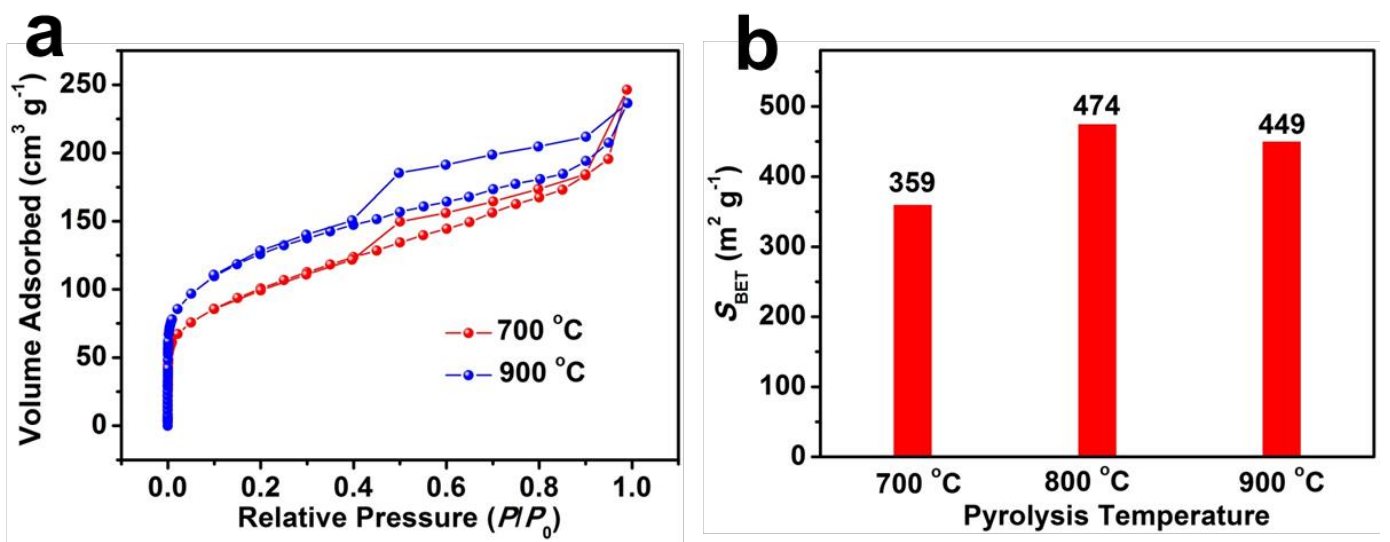

Figure S23. (a) $\mathrm{N}_{2}$ adsorption-desorption isotherms of NC@Co-NGC DSNCs obtained at 700 and $900{ }^{\circ} \mathrm{C}$, respectively. (b) BET surface area of NC@Co-NGC DSNCs obtained at various pyrolysis temperatures. 

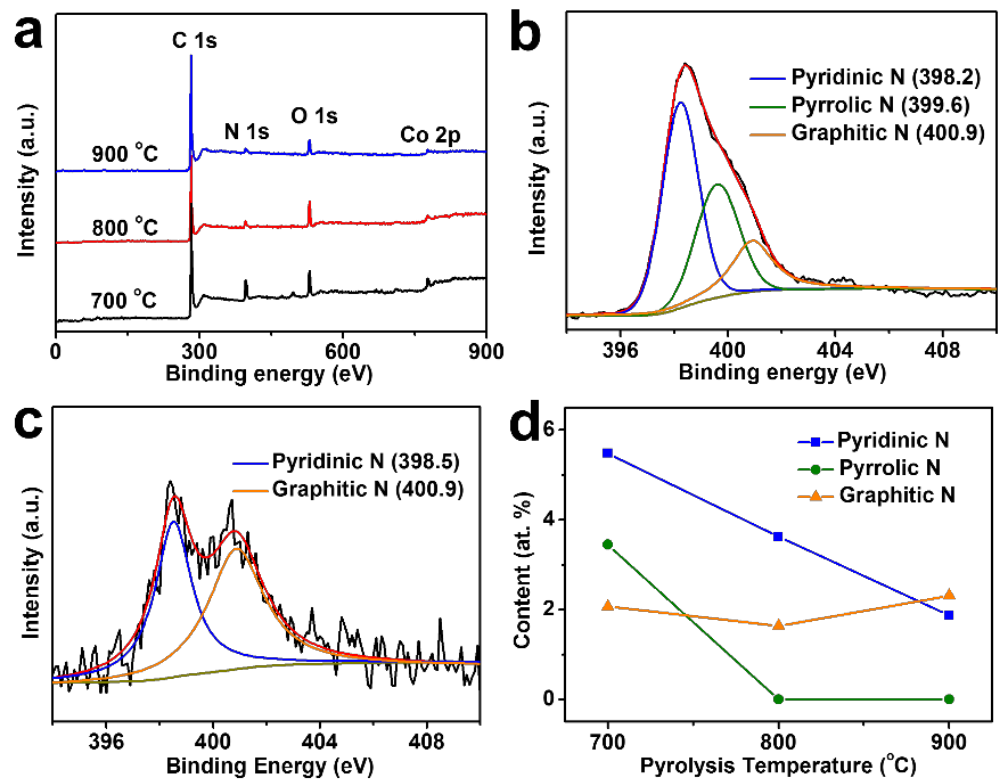

Figure S24. (a) XPS full scans of NC@Co-NGC DSNCs annealed at various temperatures. (b, c) N 1s XPS spectra of NC@Co-NGC DSNCs annealed at (b) $700{ }^{\circ} \mathrm{C}$ and (c) $900{ }^{\circ} \mathrm{C}$, respectively. (d) The content variation of pyridinic-type $\mathrm{N}$, pyrrolic-type $\mathrm{N}$ and graphitic-type $\mathrm{N}$ in NC@Co-NGC DSNCs annealed at different temperatures.
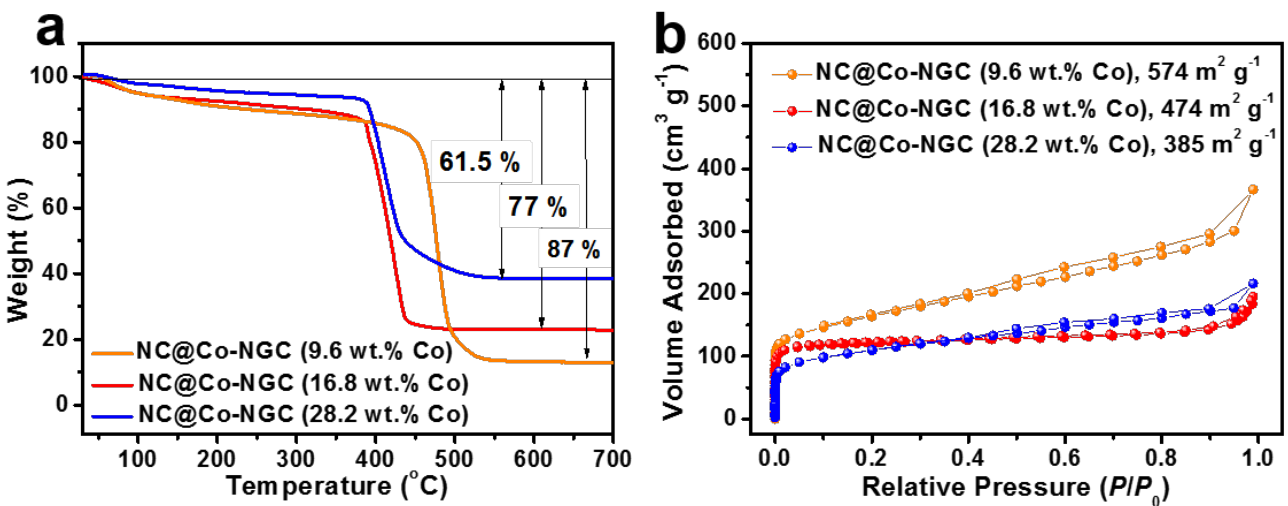

Figure S25 (a) TGA curve and (b) $\mathrm{N}_{2}$ adsorption-desorption isotherms of NC@Co-NGC DSNCs with various Co content of 9.6, 16.8 and 28.2 wt.\%. They are produced by annealing the core-shelled ZIF-8@ZIF-67 particles with different ratio of ZIF-8 and ZIF-67, which are made by tuning the initial amount of $\mathrm{Co}\left(\mathrm{NO}_{3}\right)_{2} \cdot 6 \mathrm{H}_{2} \mathrm{O}$ to 90,150 and $300 \mathrm{mg}$, respectively. 


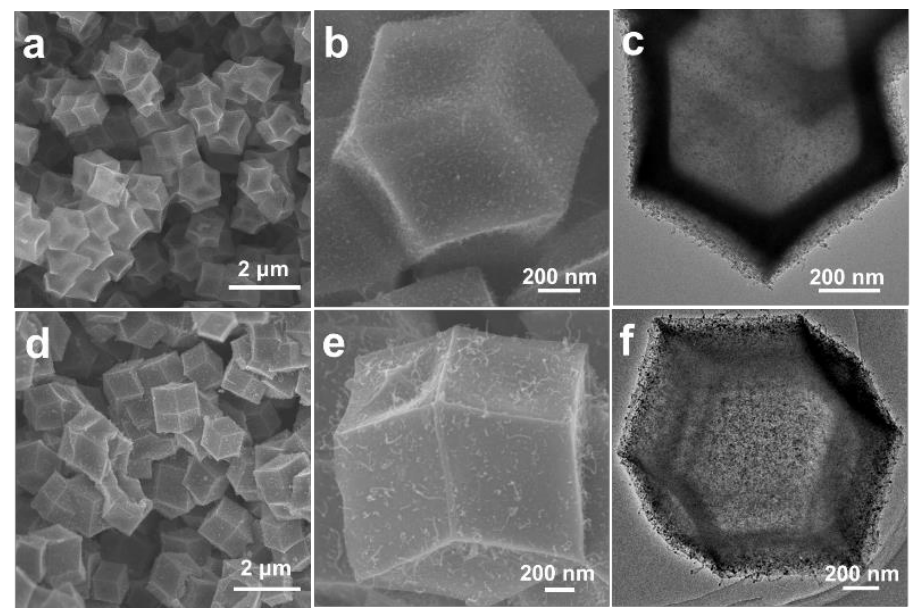

Figure S26 (a, b) FESEM and (c) TEM images of NC@Co-NGC DSNCs with Co content of 9.6 wt.\%. (d, e) FESEM and (f) TEM images of NC@Co-NGC DSNCs with Co content of 28.2 wt. $\%$.
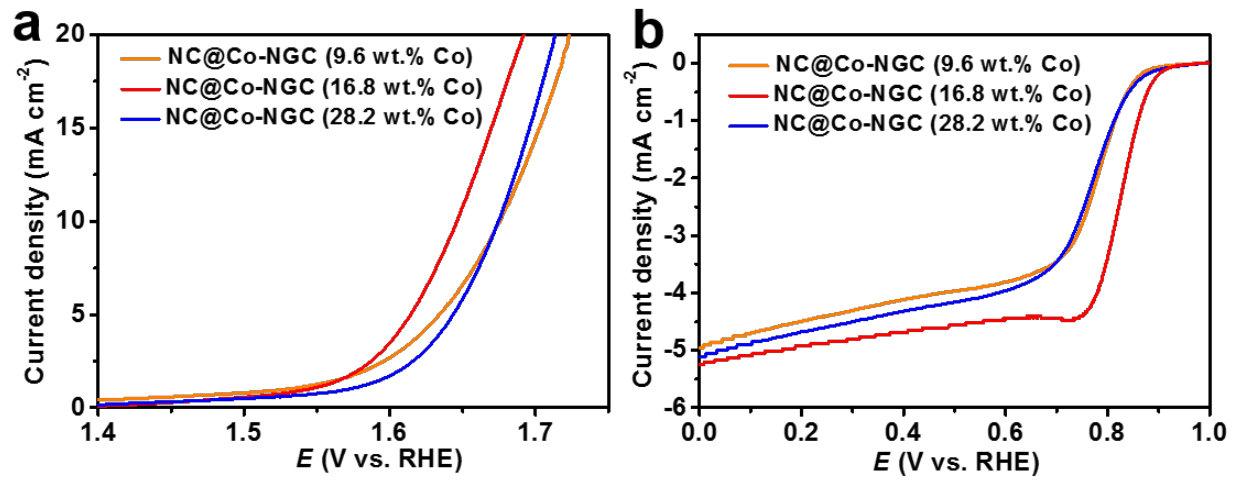

Figure S27 (a) ORR and (b) OER polarization curves of NC@Co-NGC DSNCs with Co content of 9.6, 16.8 and $23.2 \mathrm{wt} . \%$, measured at a rotation rate of $1600 \mathrm{rpm}$ in $\mathrm{O}_{2}$-saturated 0.1 $\mathrm{M} \mathrm{KOH}$ solution at a scan rate of $10 \mathrm{mV} \mathrm{s}^{-1}$. 

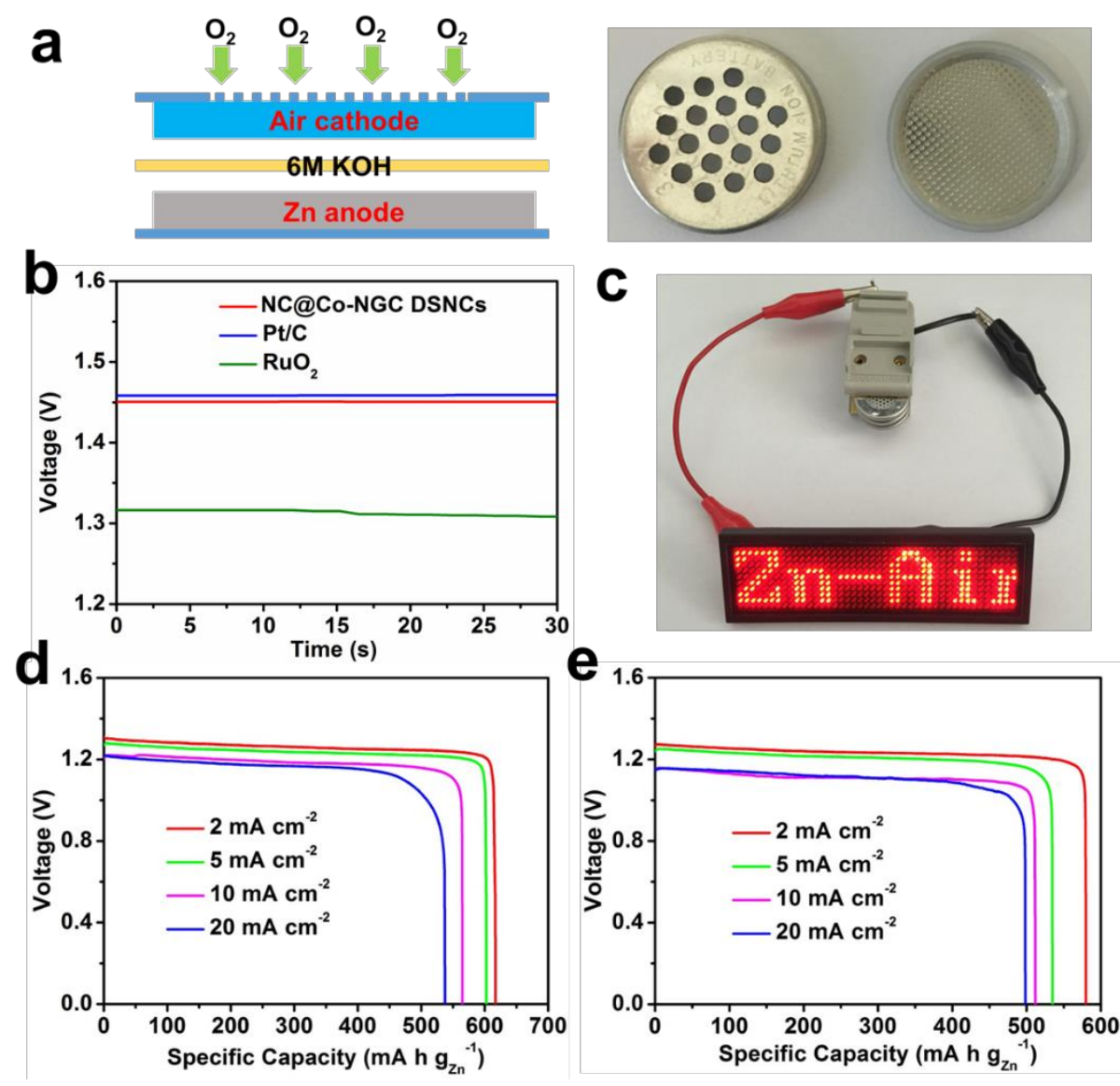

Figure S28. (a) Schematic of the primary Zn-air battery with a carbon paper casted with catalysts as air cathode, a $\mathrm{Zn}$ plate as anode and a glassy fiber membrane soaked with aqueous $\mathrm{KOH}$ electrolyte as the separator. (b) Open-circuit voltages of primary $\mathrm{Zn}$-air batteries with NC@Co-NGC DSNC, $\mathrm{RuO}_{2}$ and Pt/C catalysts, respectively. (c) Optical image of a LED panel lighted by three $\mathrm{Zn}$-air batteries in series. $(\mathrm{d}, \mathrm{e})$ Discharge curves of primary $\mathrm{Zn}$-air batteries with NC@Co-NGC DSNCs and Pt/C as cathode catalyst at various current densities of 2-20 $\mathrm{mA} \mathrm{cm}{ }^{-2}$, respectively.

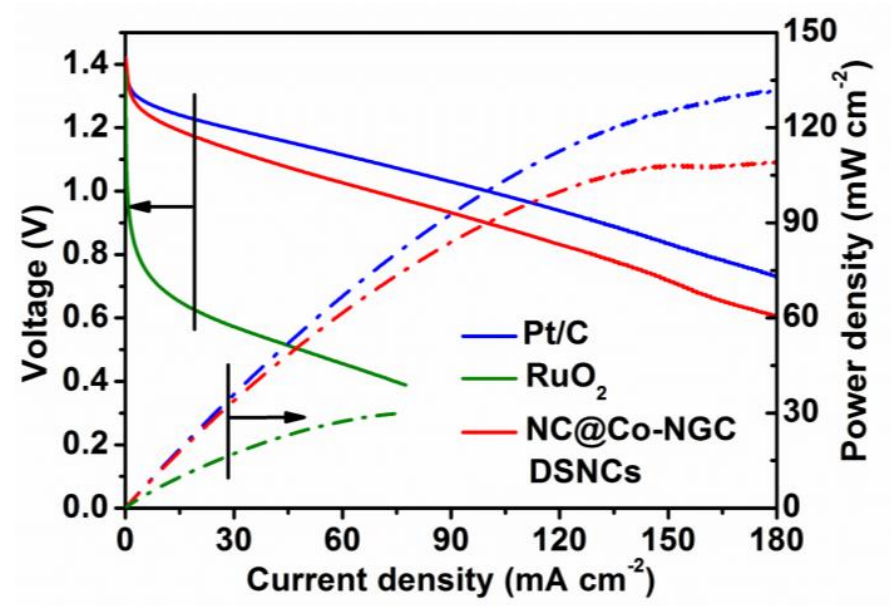

Figure S29. Discharge polarization curves and corresponding power density curves of the primary Zn-air batteries with $\mathrm{NC} @$ Co-NGC DSNC, $\mathrm{RuO}_{2}$ and $\mathrm{Pt} / \mathrm{C}$ catalysts at a scan rate of $10 \mathrm{mV} \mathrm{s}^{-1}$. 


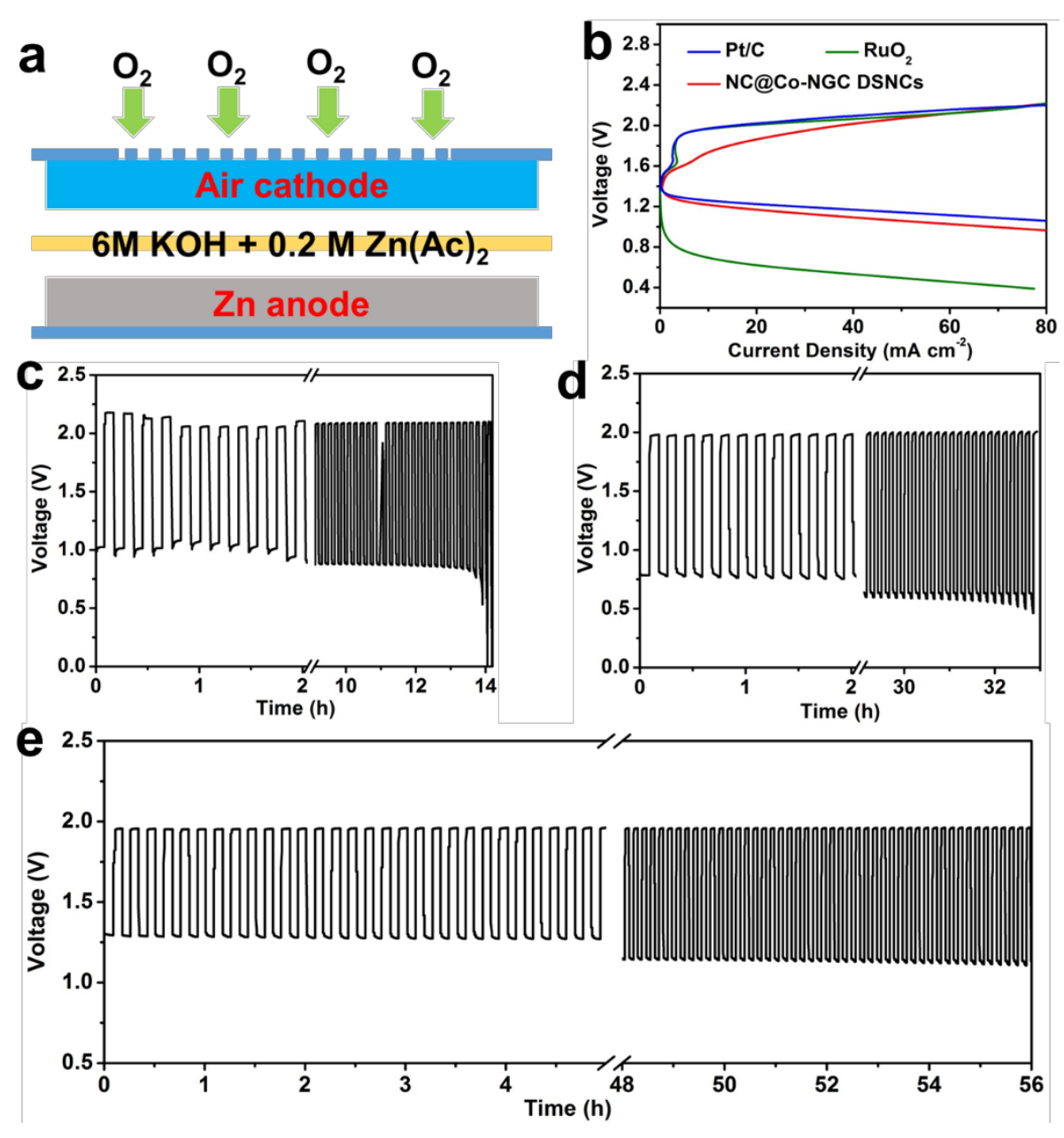

Figure S30. (a) Schematic of the rechargeable Zn-air battery. (b) Discharge-charge polarization curves of rechargeable Zn-air batteries with NC@Co-NGC DSNCs, Pt/C or $\mathrm{RuO}_{2}$ as cathode catalysts. (c-e) Galvanostatic charge-discharge cycling performance of rechargeable Zn-air batteries with Pt/C, $\mathrm{RuO}_{2}$ and $\mathrm{NC} @$ Co-NGC DSNC catalysts at a current density of $10 \mathrm{~mA}$ $\mathrm{cm}^{-2}$, respectively.
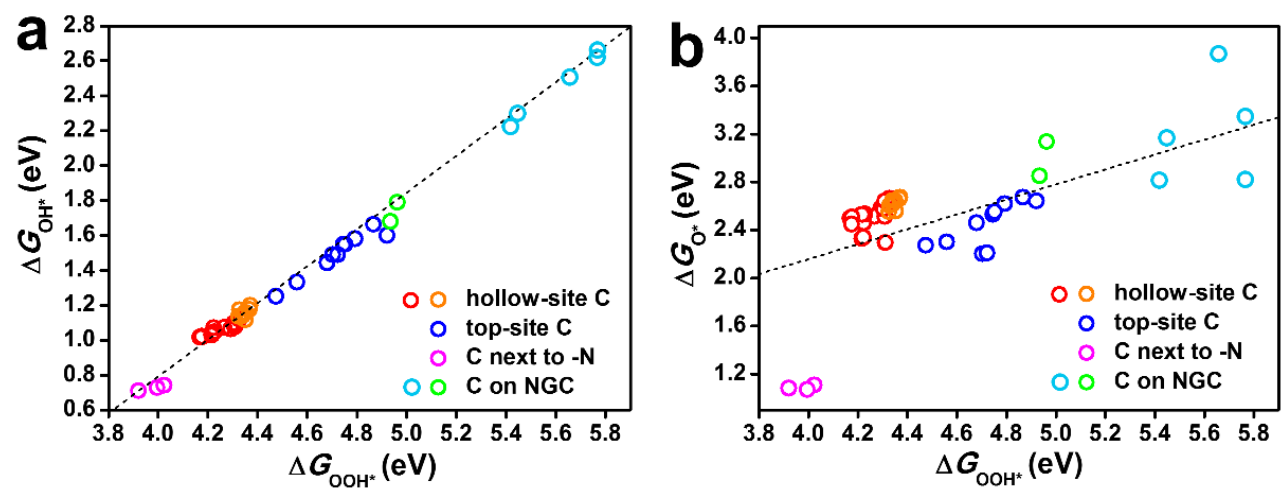

Figure S31. (a) Adsorption free energies of $\mathrm{OOH}^{*}$ versus adsorption energies of $\mathrm{OH}^{*}$ on different sites of Co-NGC for ORR. (b) Adsorption free energies of $\mathrm{OOH}^{*}$ versus adsorption energies of $\mathrm{O}^{*}$ on different sites of Co-NGC for OER. 
Table S1. A comparison on the electrocatalytic activity of the recently reported bifunctional catalysts.

\begin{tabular}{|c|c|c|c|c|c|c|c|}
\hline Catalyst & $\begin{array}{c}\text { ORR } \\
\text { onset } \\
\text { potential } \\
\text { (V vs. } \\
\text { RHE) }\end{array}$ & $\begin{array}{c}\text { ORR } \\
\text { half-wave } \\
\text { potential } \\
\left(E_{1 / 2}\right) \\
(V \text { vs. } \\
\text { RHE) } \\
\end{array}$ & 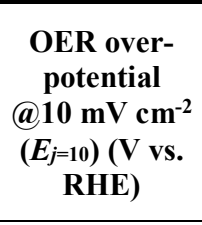 & 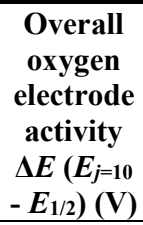 & $\begin{array}{l}\text { Catalyst } \\
\text { loading } \\
\left(\mathrm{mg} \mathrm{cm}^{-2}\right)\end{array}$ & $\begin{array}{c}\text { Electrolyt } \\
\mathrm{e}\end{array}$ & Reference \\
\hline $\begin{array}{c}\text { NC@Co-NGC } \\
\text { DSNCs }\end{array}$ & 0.92 & 0.82 & 1.64 & 0.82 & 0.4 & $\begin{array}{l}\text { 0.1 M } \\
\text { KOH }\end{array}$ & $\begin{array}{l}\text { This } \\
\text { work }\end{array}$ \\
\hline $\mathrm{RuO}_{2}$ & 0.85 & 0.61 & 1.61 & 1.00 & 0.4 & $\begin{array}{l}0.1 \mathrm{M} \\
\mathrm{KOH}\end{array}$ & $\begin{array}{l}\text { This } \\
\text { work }\end{array}$ \\
\hline $\mathrm{Pt} / \mathrm{C}$ & 0.96 & 0.82 & 1.76 & 0.94 & 0.4 & $\begin{array}{l}0.1 \mathrm{M} \\
\mathrm{KOH}\end{array}$ & $\begin{array}{l}\text { This } \\
\text { work }\end{array}$ \\
\hline $\begin{array}{c}\mathrm{N}, \mathrm{S} \text {-carbon } \\
\text { nanosheet }\end{array}$ & 0.92 & 0.77 & 1.65 & 0.88 & 0.2 & $\begin{array}{l}0.1 \mathrm{M} \\
\mathrm{KOH}\end{array}$ & [S15] \\
\hline $\begin{array}{l}\mathrm{P}-\mathrm{g}-\mathrm{C}_{3} \mathrm{~N}_{4} \text { on } \\
\text { carbon paper }\end{array}$ & 0.94 & 0.67 & 1.63 & 0.96 & $\sim 0.2$ & $\begin{array}{l}0.1 \mathrm{M} \\
\mathrm{KOH}\end{array}$ & [S16] \\
\hline $\begin{array}{c}\mathrm{Mn}_{\mathrm{x}} \mathrm{O}_{\mathrm{y}} / \mathrm{N} \\
\text {-carbon }\end{array}$ & 0.85 & 0.81 & 1.68 & 0.87 & 0.21 & $\begin{array}{l}0.1 \mathrm{M} \\
\mathrm{KOH}\end{array}$ & [S17] \\
\hline $\begin{array}{l}\mathrm{CoO} / \mathrm{N}- \\
\text { graphene }\end{array}$ & 0.90 & 0.81 & 1.57 & 0.76 & 0.7 & $1 \mathrm{M} \mathrm{KOH}$ & [S18] \\
\hline $\begin{array}{l}\mathrm{Co}_{3} \mathrm{O}_{4} / \mathrm{N}- \\
\text { graphene }\end{array}$ & 0.86 & 0.83 & 1.54 & 0.71 & 1.0 & $1 \mathrm{M} \mathrm{KOH}$ & [S19] \\
\hline $\begin{array}{c}\mathrm{MnCo}_{2} \mathrm{O}_{4} / \mathrm{N}- \\
\mathrm{CNT}\end{array}$ & 0.85 & 0.63 & 1.65 & 1.02 & 0.21 & $\begin{array}{l}0.1 \mathrm{M} \\
\mathrm{KOH}\end{array}$ & [S20] \\
\hline $\mathrm{Ti}_{3} \mathrm{C}_{2} / \mathrm{g}-\mathrm{C}_{3} \mathrm{~N}_{4}$ & 0.72 & 0.51 & 1.65 & 1.04 & 1.4 & $\begin{array}{l}0.1 \mathrm{M} \\
\mathrm{KOH}\end{array}$ & {$[\mathrm{S} 21]$} \\
\hline
\end{tabular}




\section{References}

S1. Kresse G., Furthmüller J. Efficient iterative schemes for ab initio total-energy calculations using a planewave basis set. Phys. Rev. B 54, 11169 (1996).

S2. Kresse G., Joubert D. From ultrasoft pseudopotentials to the projector augmented-wave method. Phys. Rev. $B$ 59, 1758 (1999).

S3. Perdew J.P., Burke K., Ernzerhof M. Generalized gradient approximation made simple. Phys. Rev. Lett. 77, 3865 (1996).

S4. Novoselov K.S., et al. Electric Field Effect in Atomically Thin Carbon Films. Science 306, 666-669 (2004).

S5. Ashcroft N.W. ND Mermin Solid state physics. Saunders College, Philadelphia, 120 (1976).

S6. Grimme S., Antony J., Ehrlich S., Krieg H. A consistent and accurate ab initio parametrization of density functional dispersion correction (DFT-D) for the 94 elements H-Pu. J. Chem. Phys. 132, 154104 (2010).

S7. Rossmeisl J., Qu Z.-W., Zhu H., Kroes G.-J., Nørskov J.K. Electrolysis of water on oxide surfaces. J. Electroanal. Chem. 607, 83-89 (2007).

S8. $\quad$ Li M., Zhang L., Xu Q., Niu J., Xia Z. N-doped graphene as catalysts for oxygen reduction and oxygen evolution reactions: Theoretical considerations. J. Catal. 314, 66-72 (2014).

S9. Man I.C., et al. Universality in oxygen evolution electrocatalysis on oxide surfaces. ChemCatChem 3, 11591165 (2011)

S10. Liu R., Wu D., Feng X., Müllen K. Nitrogen-doped ordered mesoporous graphitic arrays with high electrocatalytic activity for oxygen reduction. Angew. Chem. Int. Ed. 49, 2565-2569 (2010).

S11. Zheng Y., Jiao Y., Ge L., Jaroniec M., Qiao S.Z. Two-Step Boron and Nitrogen Doping in Graphene for Enhanced Synergistic Catalysis. Angew. Chem. Int. Ed. 52, 3110-3116 (2013).

S12. Reuter K., Scheffler M. Composition, structure, and stability of $\mathrm{RuO}_{2}(110)$ as a function of oxygen pressure. Phys. Rev. B 65, 035406 (2001).

S13. Stull D. Prophet, JANAF Thermochemical Tables. National Bureau of Standards, NSRDS-NBS (1971).

S14. Zhang J., Zhao Z., Xia Z., Dai L. A metal-free bifunctional electrocatalyst for oxygen reduction and oxygen evolution reactions. Nat. Nanotechnol. 10, 444-452 (2015).

S15. Qu K., Zheng Y., Dai S., Qiao S.Z. Graphene oxide-polydopamine derived N, S-codoped carbon nanosheets as superior bifunctional electrocatalysts for oxygen reduction and evolution. Nano Energy 19, 373-381 (2016).

S16. Ma T.Y., Ran J., Dai S., Jaroniec M., Qiao S.Z. Phosphorus-doped graphitic carbon nitrides grown in situ on carbon-fiber paper: flexible and reversible oxygen electrodes. Angew. Chem. Int. Ed. 54, 4646-4650 (2015).

$\mathrm{S} 17$. Masa J., et al. $\mathrm{Mn}_{\mathrm{x}} \mathrm{O}_{\mathrm{y}} / \mathrm{NC}$ and $\mathrm{Co}_{\mathrm{x}} \mathrm{O}_{\mathrm{y}} / \mathrm{NC}$ Nanoparticles Embedded in a Nitrogen-Doped Carbon Matrix for High-Performance Bifunctional Oxygen Electrodes. Angew. Chem. Int. Ed. 53, 8508-8512 (2014).

S18. Mao S., Wen Z., Huang T., Hou Y., Chen J. High-performance bi-functional electrocatalysts of 3D crumpled graphene-cobalt oxide nanohybrids for oxygen reduction and evolution reactions. Energy Environ. Sci. 7, 609-616 (2014).

$\mathrm{S} 19$. Liang $\mathrm{Y}$., et al. $\mathrm{Co}_{3} \mathrm{O}_{4}$ nanocrystals on graphene as a synergistic catalyst for oxygen reduction reaction. Nat. Mater. 10, 780-786 (2011).

S20. Zhao A., et al. Spinel Mn-Co Oxide in N-Doped Carbon Nanotubes as a Bifunctional Electrocatalyst Synthesized by Oxidative Cutting. J. Am. Chem. Soc. 136, 7551-7554 (2014).

S21. Ma T.Y., Cao J.L., Jaroniec M., Qiao S.Z. Interacting Carbon Nitride and Titanium Carbide Nanosheets for High-Performance Oxygen Evolution. Angew. Chem. Int. Ed. 55, 1138-1142 (2015). 